\title{
Berieht über die Privatklinik des Herrn Professor Dr. Hoeftman in Königsberg Pr.
}

Von

Gustaf Scheiutz.

Yit 68 Abbildungen im Text.

Seitdem Dr. Strube im Jahre 1900 einen Bericht über die Ho eft mansche Privatklinik veröffentlichte, ist kein zweiter erschienen. Wenn ich mich nun an einen solchen herangemacht und den Zeitraum für denselben soweit gesetzt habe wie zehn Jahre, bin ich mir wohl bewusst, dass dieser Bericht keineswegs erschöpfend sein kann. Dazu ist das Material viel zu gross und reichhaltig.

(Allein in der Klinik stationär behandelt wurden in dieser Zeit $12718 \mathrm{~Pa}$ tienten.) Es können daher nur einzelne Fälle berausgegriffen oder kurze Ausführungen über die hauptsächlich zar Verwendung

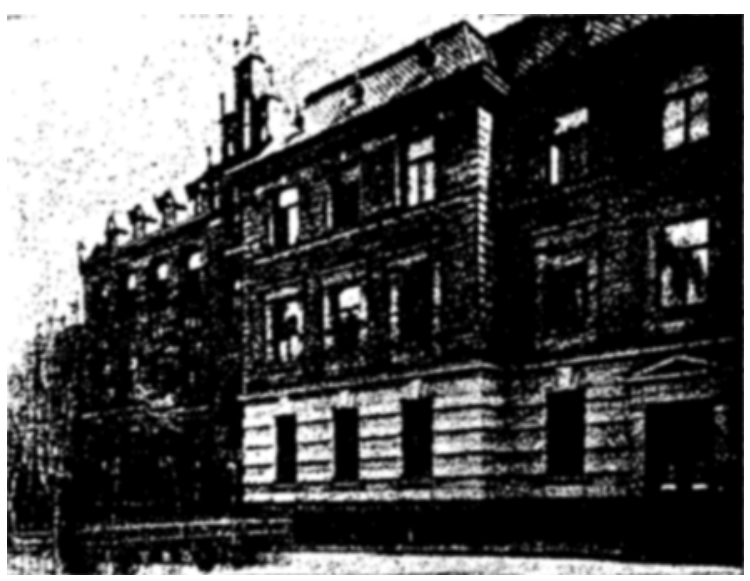

Fig. 1.

Ansicht der Klinik von der Strassenseite. kommenden Behandlungsmethoden gemacht werden, um einen Überblick zu geben.

Zunächst mögen einige allgemeine Notizen vorangeschickt werden.

Die Klinik ist im Jahre 1882 gegründet und hat sich aus kleinen Anfängen allmählich zu ibrer jetzigen Grösse ausgewachsen. Mit ihren 120 Betten ist sie wohl zurzeit die grösste Privatklinik Deutschlands.

Sie besteht aus zwei Gebäuden (Fig. 1 r. 2), eins für die eigentlicbe Klinik, das andere für das damit verbundene Zanderinstitut. 


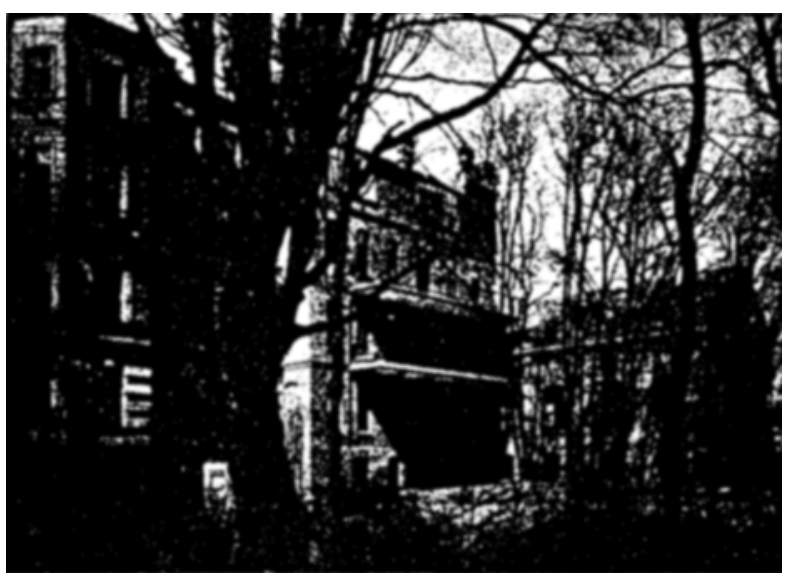

Fig. 2.

Ansicht der Klinik von der Gartenseite.

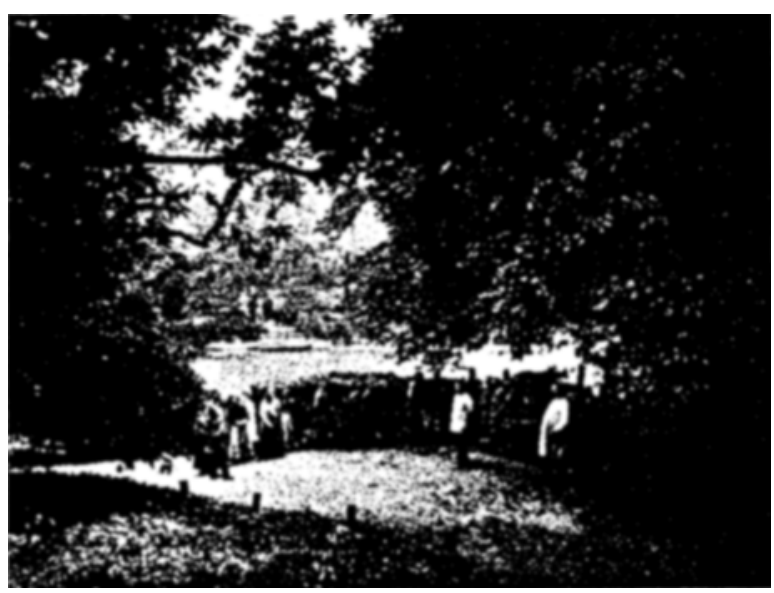

Fig. 3

Partie vom Garten an Schlossteich.

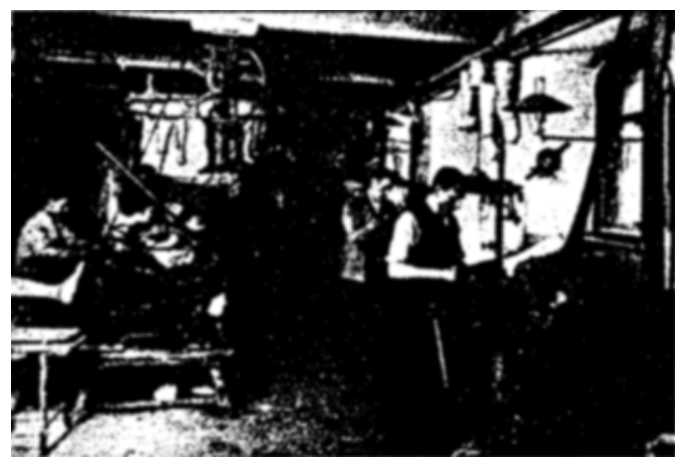

Eig. 4.

Orthopüdische Werkstatt.
Das Klinikgebüude ist ein dreistöckiges Haus mit Souterrain und zeigt eine Strassenfront von 53 Metern. An seine Rückseite schliesst sich ein grosser Garten mit einem Flächeninhalt von 3966 Quidratmeter, der sich bis zu dem Schlossteich hinstreckt (Fig. 3), wollurch den Patienten auch die Möglichkeit des Kahnfahrens geboten wird.

In der Mitte des Hauses führt eine bequeme, in Absützen gebaute Treppe zn den verschiedenen Stockwerken.

Ausserdem geht durch sämtliche Etagen ein Fahrstuhl.

Im Souterrain befinden sich die Küche und die sonstigen Wirtschaftsräume. Ausserdem aber ist hier die orthopädische Werkstatt (Fig. 4) und die Badeanstalt untergebracht. Letztere ist auch für Auswärtige offen und es können hier alle möglichen Arten ron Bädern verabreicht werden.

Unter dem Souterrain liegen noch tiefere Kellerräume. Hier stohen die vier grossen Kessel für die Zentralheizung.

Im Erdgeschoss befinden sich ausser der Privatwobnung für den Inhaber und Wohnung für zwei Assistenzärzte die Sprechzimmer (Fig. 万), Wartezimmer und Büroräume etc. 
(Fig. 6). Ausserdem ist die Röntgeneinrichtung hier untergebracht') (Fig. 7).

Die eigentlichen Krankenzimmer, dreissig an der Zabl, liegen in erster, zweiter und dritter Etage und zeigen, je nach der Klasse, verschiedene Ausstattung und Grösse, von kleineren Räumen bis zu grossen Salons mit drei Fenstern und Balkon.

Im ersten Stock ist ausserdem die Kinderstation eingeräumt. Diese besteht ans einem grossen Saal für kleine Kinder sowie einzelne Räume für grössere Knaben und Miidchen.

Entsprechend den Räumlichkeiten der Kinderstation befindet sich im zweiten Stock ein Schlafsaal mit fünfundzwanzig Betten mit einem daneben liegenden Tagesraum für Lnfallverletzte berechnet, die auf Kosten der Berufsgenossenschaften behandelt werden. Sowohl dieser Männersaal wie die Kinderstation haben grosse Balkons, die den Autenthalt in der freien Luft gestatten.

In der dritten Etage liegt der Operationssaal und daneben ein photograpbisches Atelier; beide mit Oberlicht und Seitenlicht versehen.

Die photographische Abteilung wird sehr fleissig gebraucht, da die Unfallverletzten in der Regel sowohl bei der Aufnahme wie bei der Entlassung photographiert werden, um einen objektiven Beweis für die erzielte Besserung liefern zu können. Es ist dies wohl ein ziemlich kostspieliges Verfabren, das sich aber gut bewährt.

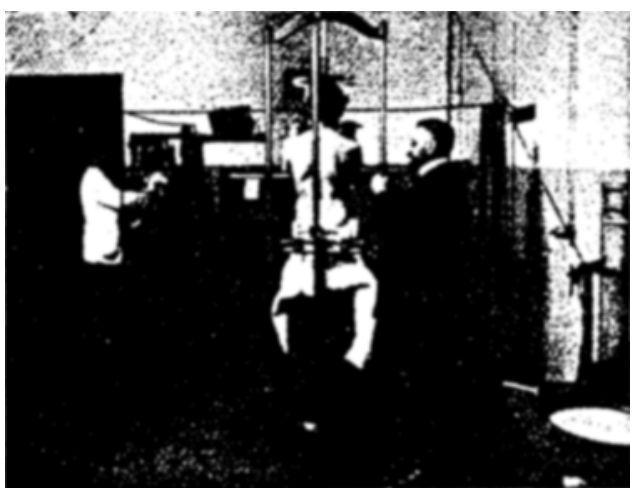

Fig. 6.

Zimmer fär Messungen, Elektrisieren etc.

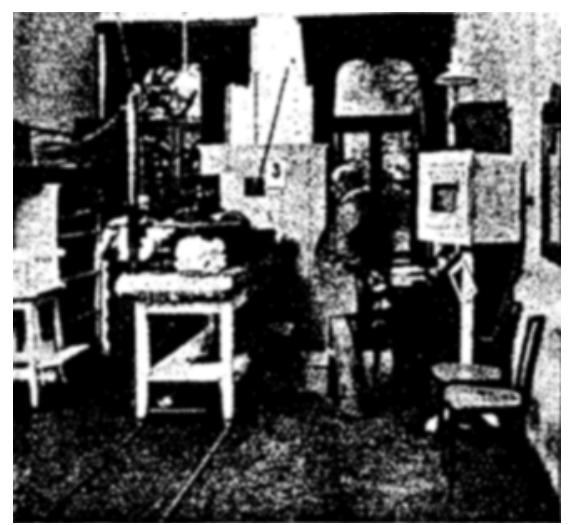

Abb. 7.

Röntgenzimmer.

1) Soit 1904 wird dio Röntgenabteilung von Herrn Dr. Mattbias geleitet, der die Untersuchungen für eigene Rechnung ausführt. 
In einem hesonderen Anbal ist das Zander-Institut (Fig. 8 u. 9) untergebracht. Der Saal ist 27 Neter lang, 11 Meter breit und 7\% Meter hoch, mit achtzehn Fenstern versehen und enthält siebenundsechzig Apparate. In der Mitte steht ein grosses, mit einem Springbrunneu rersehenes Zementbassin,

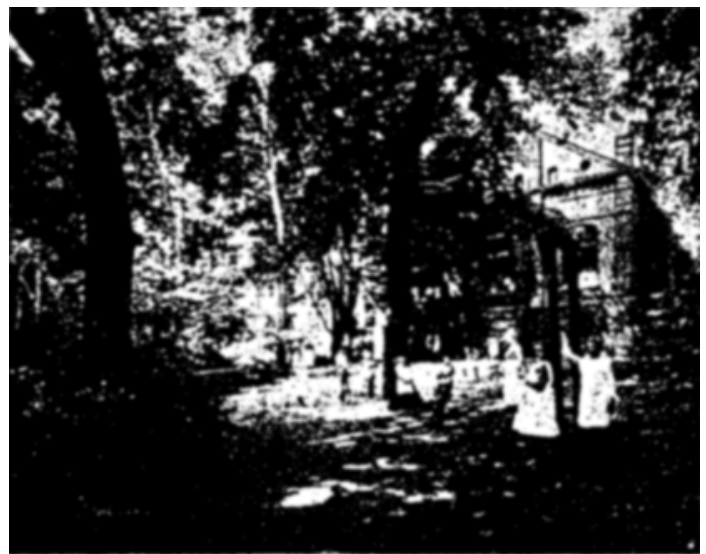

Fig 8.

Kinderspielplatz. Im Hintergrunde das Zander-Institut. um zu verhüten, dass die Luft trocken wird. Die Apparate für die passiven Bewegungen werden durch einen elektrischen Motor betrieben, der ebenso wie die Wellen und Riemenleitungen im Keller sich befindet, was für die Sicherheit des Betriebes sebr wesentlich ist.

Seit der Gründung der Klinik sind bis jetzt ${ }^{1}$ ) 22637 Patienten stationär behandelt worden. Vom 1. Januar 1903 bis 1. Januar 1913 beträgt die Zahl der in der Klinik aufgenommenen Patienten 12718 ; die wäbrend derselben Zeit im Zander-Institut behandelten 8933, wobei jedoch zu bemerken ist, dass der grössere Teil der in Zander-Institut Behandelten aus klinischen Patienten besteht.

Zu einem so grossen Betrieb geliört selbstredend auch ein grosses Personal. So z. B. arbeiten allein in der orthopädischen Werkstatt, trotz des

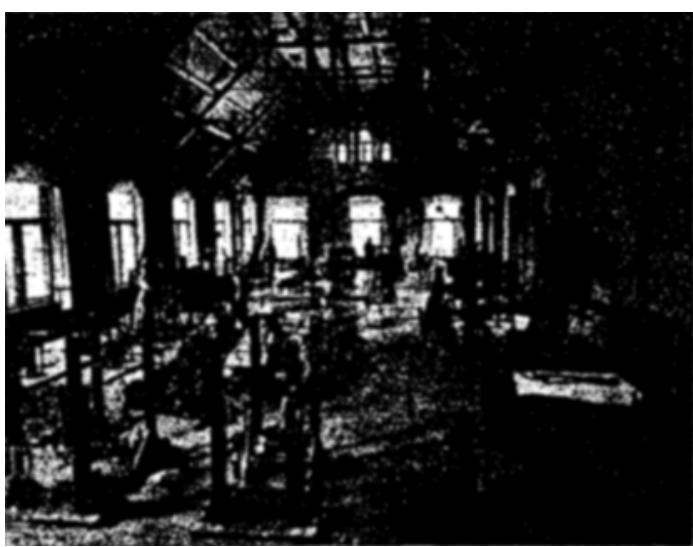

Fig. 9.

Zander-Institut. elektrischen Betriebes derselben, zurzeit zwölf Personen. Im Durchschnitt ist in der ganzen Kilinik ein Personal ron sechzig Personen beschäftigt.

\section{Statistik.}

Die Zahl der in der Klinik behandelten Patienten setzt sich teils aus Privatpatienten, teils aus solchen zusammen, die ron der Landesversicherungsanstalt, den Berufsgenossenschaften und den Krankenkassen zur Behandlung oder Begutachtung überwiesen werden. Die Zahl der Unfallverletzten ist daher besonders gross. Das Krankenmaterial beschränkt sich also nicht nur auf rein orthopädische Fälle, sondern umfasst beinah das ganze Gebiet der Medizin, was 
Eericht über die Privatklinik des Herro Professor Dr. Hoeftra an in Königsberg Pr. 13

auch darauf zurückzuführen ist, dass andere Ärzte, sowohl aus der Stadt, wie aus der Provinz ihre Patienten hineinschicken, um sie in dauernder Behandlung und Beobachtung zu wissen.

Eine genaue Rubrizierung der Krankheitsfälle würde daher alle möglichen Gebiete der Medizin umfassen müssen und sehr viel Raum beanspruchen. Es sollen daher nur einige Rubriken berücksichtigt werden, um eine Übersicht zu geben. Unter den 12700 während der zebn Jahre in der Klinik stationär behandelten Patienten waren folgende Fälle:

\begin{tabular}{|c|c|c|c|c|c|c|c|c|c|c|c|}
\hline & 19 & & 190 & 19 & 1907 & 1908 & 1909 & 91910 & 1914 & 1912 & $\underset{\substack{\text { Zu- } \\
\text { sammien }}}{ }$ \\
\hline Spondylitis & 79 & 75 & 77 & 71 & 76 & 72 & 66 & 76 & 98 & 76 & 766 \\
\hline Skoliosen & 62 & 61 & 80 & 58 & 50 & 69 & 45 & 70 & 61 & 80 & 636 \\
\hline Tuberkulöse Gelenkerkrankungen & 31 & 36 & 64 & 70 & 68 & 47 & 75 & 69 & 83 & 101 & 644 \\
\hline Kongenitale Luxationen . . . & 3 & 10 & 13 & 10 & 14 & 12 & 12 & 14 & 15 & 8 & 111 \\
\hline Spinale Kinderlähmungen & 6 & 2 & 5 & 7 & 6 & 7 & 14 & 23 & 25 & 38 & 133 \\
\hline Andere Lāhmungen . & 15 & 16 & 21 & 24 & 27 & 38 & 41 & 47 & 54 & 45 & 328 \\
\hline D-Beine . . . . & 4 & 4 & 1 & - & - & 4 & 5 & 9 & 9 & 3 & 39 \\
\hline X-Beine . . & 2 & 4 & 3 & 4 & 4 & 12 & 12 & 7 & 14 & 12 & 73 \\
\hline Klumpfusse & 10 & 8 & 17 & 9 & 3 & 26 & 25 & 24 & 21 & 22 & 165 \\
\hline Plattfüsse. . & 12 & 14 & 28 & 22 & 31 & 52 & 55 & 63 & 50 & 63 & 390 \\
\hline Spitzfüsse. . & - & 7 & - & 1 & 1 & 5 & 11 & 11 & 8 & 8 & 52 \\
\hline Rheumatisehe Erkrankungen & 27 & 32 & 33 & 26 & 66 & 58 & 56 & 58 & 46 & 65 & 467 \\
\hline $\begin{array}{l}\text { Hysterio und Neurosen. } \\
\text { Erkrankangen der Respirations. }\end{array}$ & 35 & 29 & 37 & 28 & 29 & 18 & 25 & 12 & 19 & 21 & 253 \\
\hline und Zirkulations-Organe & 18 & 15 & 19 & 20 & 10 & 21 & 25 & 19 & 11 & 10 & 168 \\
\hline 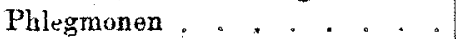 & 13 & 9 & 13 & 7 & 7 & 16 & 46 & 52 & 50 & 51 & 264 \\
\hline ungen & 4 & 4 & 3 & 5 & 3 & 5 & 8 & 8 & 12 & 7 & 59 \\
\hline Dsteomyelitis. & 5 & 7 & 9 & 4 & 10 & 19 & 13 & 17 & 29 & 11 & 124 \\
\hline rüche & & & & & & & & & & & \\
\hline ls & 4 & 2 & 1 & 1 & 3 & 4 & 4 & 10 & 8 & 9 & 46 \\
\hline des & 1 & - & $\ldots$ & - & - & $\div$ & 2 & 3 & 1 & 1 & 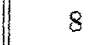 \\
\hline lsäule. & 4 & 10 & 15 & 8 & 13 & 31 & 38 & 43 & 38 & 48 & 248 \\
\hline $\operatorname{des} B$ & 2 & 4 & 7 & 4 & 5 & 7 & 4 & 5 & 11 & 10 & 59 \\
\hline elbalses & 1 & 3 & 15 & 11 & 8 & 11 & 19 & 25 & 27 & 23 & 15 \\
\hline des Oberschenkels. & 18 & 11 & 18 & 19 & 14 & 26 & 44 & 36 & 45 & 28 & 259 \\
\hline der Knie & 2 & 3 & 4 & - & 4 & 2 & 11 & 9 & 8 & 7 & 50 \\
\hline des Unterschenkels & 41 & 44 & 75 & 32 & 96 & 108 & 125 & 149 & 160 & 172 & 1022 \\
\hline der Fussknochen . & 2 & 4 & 10 & 2 & 11 & 12 & 12 & 17 & 14 & 25 & 109 \\
\hline terblattes & 1 & 1 & 8 & 2 & - & 2 & 3 & 4 & 2 & 3 & 26 \\
\hline solbeines. & 5 & 3 & 10 & 5 & 8 & 15 & 10 & 9 & 11 & 7 & 83 \\
\hline nes & 8 & 6 & 30 & 19 & 23 & 26 & 31 & 24 & 34 & 14 & 215 \\
\hline bogens. & 7 & 7 & 9 & 4 & 4 & 11 & 5 & 12 & 8 & 9 & 76 \\
\hline des Unterarmes & 22 & 19 & 29 & 19 & 17 & 34 & 46 & 40 & 34 & 36 & 296 \\
\hline der Handwarzelknoeben & - & - & $2>>$ & - & - & 2 & 4 & 6 & 4 & 5 & 24 \\
\hline der Finger & 3 & - & 2 & 3 & 3 & 5 & 5 & $3\}$ & 10 & 3 & 37 \\
\hline der Rippen . & 6 & 3 & 5 & 5 & 6 & 13 & 6 & 6 & 12 & 6 & 68 \\
\hline erren & & & & & & & & & & & \\
\hline & 10 & 6 & 8 & 0 & 8 & 34 & 41 & 27 & 29 & 27 & 196 \\
\hline des Ellenbogengelenkes & 5 & 1 & 3 & 4 & 6 & 7 & 10 & 16 & 6 & 8 & 66 \\
\hline
\end{tabular}




\begin{tabular}{|c|c|c|c|c|c|c|c|c|c|c|c|}
\hline & 1903 & 1904 & 1905 & 1906 & 1907 & 1908 & 1909 & 1910 & 1911 & 1912 & $\underset{\text { sammien }}{\mathrm{Zu}^{-}}$ \\
\hline \multicolumn{12}{|l|}{ Verenkungen: } \\
\hline $\begin{array}{l}\text { der Finger. } . \cdot . \\
\text { des Hüftgelenkes (nicht kon- }\end{array}$ & - & 2 & - & - & -1 & - & - & 4 & 3 & 2 & 11 \\
\hline genitale) & - & 1 & - & 2 & 2 & 4 & $-\cdots$ & 4 & 3 & 2 & 18 \\
\hline \multicolumn{12}{|l|}{$\begin{array}{l}\text { Quetschungen u. Verstauchungen: } \\
\text { von Kopf, Wirbelsäule und }\end{array}$} \\
\hline Rucken . . . . . . & 16 & 16 & 13 & 14 & 32 & 41 & 39 & 23 & 42 & 31 & 267 \\
\hline der Brust & 2 & 6 & 6 & 3 & 4 & 13 & 11 & 12 & 8 & 13 & 78 \\
\hline $\begin{array}{l}\text { des Onterleibes. } \\
\text { von Becken, Hüfte und Ober- }\end{array}$ & -1 & - & -1 & 3 & - & 3 & 3 & 1 & 1 & 5 & 16 \\
\hline schenkel. . . . . . & 7 & 6 & 11 & 7 & 18 & 12 & 9 & 16 & 11 & 14 & 111 \\
\hline von Knie und Unterschenkel & 15 & 11 & 7 & 14 & 37 & 46 & 49 & 45 & 55 & 54 & 333 \\
\hline deg Fusses . . . . . & 4 & 9 & 11 & 4 & 14 & 14 & 14 & 25 & 25 & 19 & 139 \\
\hline des Armes und der Hand & 11 & 15 & 25 & 25 & 28 & 48 & 47 & 57 & 78 & 69 & 403 \\
\hline \multicolumn{12}{|l|}{ Versteifungen: } \\
\hline des Sch & 34 & 31 & 13 & 28 & 47 & 73 & 81 & 70 & 94 & 73 & 544 \\
\hline des Ellenbogengelenkes & 5 & 6 & 7 & 4 & 10 & 20 & 14 & 31 & 26 & 34 & 157 \\
\hline der Hand- und Fingergelenke & 31 & 18 & 16 & 26 & 44 & 65 & 103 & 104 & 122 & 122 & 651 \\
\hline des Hüftgelenkes . . . . & $1)$ & 5 & 1 & - & 3 & 13 & 24 & 34 & 28 & 18 & 127 \\
\hline des Kniegelenkes . . . & 5 & 6 & 5 & 8 & 14 & 23 & 34 & 41 & 54 & 51 & 241 \\
\hline des Fussgelenkes . . . & 15 & 17 & 28 & 19 & 36 & 40 & 47 & 56 & 68 & 67 & 393 \\
\hline \multicolumn{12}{|l|}{ Verlust: } \\
\hline des Arme & -1 & - & 2 & 1 & 2 & 3 & 12 & 43 & 25 & 27 & 115 \\
\hline der Hand & -1 & - & - & - & - & - & 5 & 8 & 15 & 10 & 38 \\
\hline der Finger. & 8 & 4 & 7 & 6 & 5 & 17 & 29 & 31 & 45 & 42 & 194 \\
\hline des Beines . . & 11 & 5 & 7 & 10 & 13 & 39 & 40 & 69 & 50 & 61 & 305 \\
\hline des Fusses . . & -1 & - & 3 & 1 & 4 & 9. & 13 & 15 & 7 & 7 & 59 \\
\hline \multicolumn{12}{|l|}{ Es wurden angefertigt: } \\
\hline Gip & 225 & 218 & 213 & 238 & 240 & 286 & 215 & 269 & 256 & 218 & 2378 \\
\hline gkor & 58 & 63 & 71 & 68 & 56 & 61 & 40 & 49 & 82 & 57 & 665 \\
\hline Filzkorsetts & 18 & 27 & 28 & 37 & 28 & 47 & 48 & 36 & 41 & 38 & 348 \\
\hline Stützapparate & 55 & 28 & 71 & 62 & $5 j$ & 60 & 65 & 90 & 89 & 90 & 605 \\
\hline Ktinstliche Beine & 4 & 4 & 2 & 8 & 3 & 20 & 26 & 38 & 30 & 26 & 161 \\
\hline Stelzfusso. . . & 4 & 2 & 3 & 2 & 6 & 12 & 10 & 15 & 20 & 16 & 90 \\
\hline Kopfotitzen . . . . . & - & 1 & 2 & 1 & 2 & 2 & 3 & 7 & 7 & 2 & 27 \\
\hline Armprothesed . . . & - & - & 1 & 1 & 1 & 1 & 14 & 55 & 46 & 55 & 174 \\
\hline Plattfusssohlen . . & 110 & 92 & 143 & 148 & 178 & 173 & 226 & 292 & 276 & 308 & 1946 \\
\hline
\end{tabular}

Es wurden in den zehn Jahren 1732 Operationen bier vorgenommen, die sich etwa gleichmässig auf' die einzelnen Jahre verteilen.

Die Zahl der in den zehn Jahren ambulant behandelten Fälle dürfte annähernd die der stationären gleichkommen. So wurden z. B. im Zander-Institut rom 1. Januar 1903 bis 1. Januar 1913749 Skoliosen behandelt. 
Bericht uber die Plivatklinik des Herrn Professor Dr. Hoeftman in Königsberg Pr. 15

Aus dieser Statistik geht herror, dass im allgemeinen die Zahl der Krankheitsfälle von Jahr zu Jahr gleichmässig zugenommen hat. Für die Prothesen aber zeigt sich, dass in den ersten fünf Jahren nur wenig künstiche Beine, und so gut wie gar keine Arm-Prothesen angefertigt wurden. Vor 5 Jahren trat dann eine sprunghafte Steigerung ein, so dass die meisten Prothesen in den letzten Jahren angefertigt worden sind.

\section{Prothesen.}

Es gibt nicht viele Institute in Deutschland, wo Apparate als Ersatz für verlorene Glieder unter äratlicher Leitung angefertigt werden und wohl in keiner Anstalt dürfte das Material so mannigfaltig und reichhaltig sein wie in der hiesigen Privatklinik. Es liegt dies woll vor allem duran, dass Herr Professor Hoeftman der Anfertigung von Prothesen ein ganz besonderes Interesse entgegenbringt und auf diesem Gebiet Resultate erzielt, die woll kaum anderswo erreicht werden. Es würde sich daher wohl verlohnen, über die Konstruktion der Prothesen, wie sie bier angefertigt werden, eingehend zu berichten. Eine derartige Darstellung würde aber weit über den Rahmen dieses Berichts hinausgehen. Es kann daher nur in grossen Zügen eine allgemeine Übersicht gebracht werden.

Als allgemeines Prinzip geht Herr Professor $\mathrm{H}$ o ef $\mathrm{tm}$ an ron der Erwägung aus, weniger solche Prothesen anzufertigen, die nur den Zweck haben, den Verlast eines Gliedes zu verbergen, als vielmehr solche Apparate zu konstruieren, mit denen die Patienten etwas leisten können. Wenn irgend möglich sollen die Verletzten soweit gebracht werden, dass sie wirklich arbeiten können.

Was die Technik bei der Anfertigung der Prothesen anlangt, heisst es zunächst ein möglichst genaues Modell zn bekommen. Es wird daber ein Gipsabguss des erbaltenen Stumpfes angefertigt, wobei vorspringende Sehnenzüge, Knochenzacken oder sonst dem Druck ausgesetzte Stellen durch Anzeichnen mit Tusche kenntlich gemacht werden. Ausserdem wird stets, um die richtigen Grössenverhältnisse herauszubeliommen, von der Extremität der andern Seite, wenn diese erhalten ist, eine Konturzeichnung angefertigt. An dem tertigen Modell wird dann die Lage der Gelenke angezeichnet, und der Apparat in der orthopädischen Werkstatt in Roharbeit hergestellt.

Darauf erfolgt die erste Anprobe an dem Patienten selbst, wobei die zukiunftige Form der Prothese genau bezeichnet wird und eventuell notwendige Änderungen vorgenommen werden. Vielleicht muss noch eine zweite oder dritte Anprobe stattfinden, ehe der Apparat endgültig fertig gearbeitet werden kann.

Trotzdem kann es passieren, dass die Prothese nicht gut sitzt, und es müssen dann noch nachträglich Inderungen rorgenommen werden, bis der Apparat tadellos passt. Hierin liegt gerade die Quintessenz der ganzen Sache, denn es handelt sich manchmal um die Korrektur so geringfiigiger Fehler, dass sie einem Uneingeweihten als belanglos erscheinen mögen, deren Abhilfe jedoch für den guten Sitz und die Gebrauchsfähigkeit des künstlichen Gliedes von grösster Bedeutung ist. 
Als Material für die Apparate werden in der Hauptsache Hülsen aus Leder mit Stahlschienen und eisernen Ansatzstïcken verwandt. In einzelnen Fällen werden die Hülsen aus Filz hergestellt, der dann durch Eintränken mit Schellack die nötige Tragfähigkeit erhält. Für Stelzbeine kommt selbstverständlich auch Holz zur Verwendung. Die Fussstücke werden aus Stahlblech oder aus Holz, zum Teil aus Kork angefertigt.

Die Prothesen lassen sich naturgemäss in zwei grosse Gruppen einteilen: Beinprothesen und Armprothesen.

Um dann mit den einfachsten - den Stelzfïssen - anzufangen, werden diese je nach der Länge des Stumpfes aus einer den Oberschenkel umfassenden eventuell sich gegen das Becken stützenden Hülse und der Stelze zusammen-

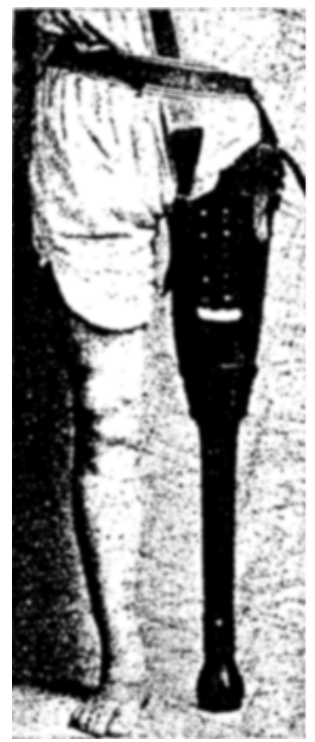

Fig. 10.?

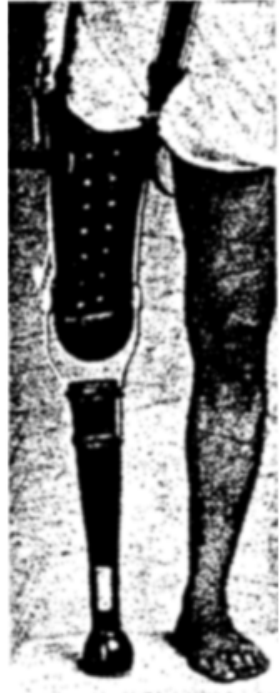

Fig. 11.

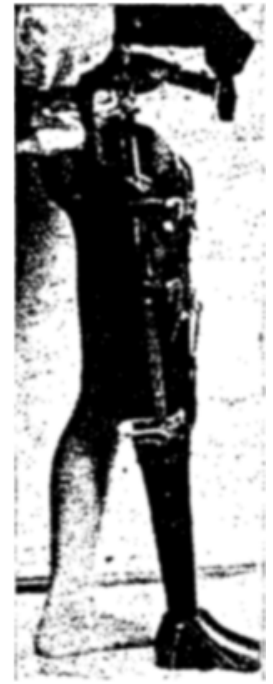

Fig. 12.

gesetzt (Fig. 10). Sie werden, wenn dies zweckmässig erscheint, mit oinem beweglichen Kniegelenk versehen, um das Sitzen bequemer zu machen (Fig. 11). Das Gelenk ist eventuell mit einem Schnepper versehen, so dass es in gestreckter Stellung nicht selbsttätig einknicken kann. Die Stelze selbst bekommt manchmal einen Holzfuss, über welchen der Patient einen Schuh überziehen kann (Fig. 12), wodurch ein natürliches Aussehen erreicht und der Gang ein sicherer wird.

Die Konstruktion der künstlichen Beine schliesst sich der der Stelzfüsse an. Nur ist hier auch der Unterschenkelteil aus einer Lederhülse mit Seitenschienen hergestellt.

Das exzentrisch angebrachte Kniegelenk (Fig. 13) ist bei solchen Apparaten fast immer beweglich. Das Strecken des Unterschenkels wird, wenn dies erforderlich ist, durch einen an der Vorderseite angebrachten Gummizug unterstützt (Fig. 14). 
Früher wurden auch die Fussgelenke regelmässig beweglich, und durch vorn und hinten angebrachte Gummipuffer federnd gemacht. Seit einigen Jahren werden sie jedoch steif gearbeitet, da es sich erwiesen hat, dass die Patienten hiermit sogar sicherer gehen, als mit beweglichen Fussgelenken, weil sie mehr Halt haben. Ausserdem fällt die bäufige Neuanschatfung der Gummipuffer fort $^{1}$ ).

Für ein sicheres Gehen mit steifen Fussgelenken ist aber erforderlich, dass die künstliche Fusssohle nicht plan, sondern leicht nach vorne und nach hinten aufgebogen ist, damit der Fuss beim Gehen regelrecht abgewickelt werden kann. Sie darf auch nicht länger sein als bis zum Metatarsopbalangeal-Gelenk der grossen Zehe eines normalen Fusses. Das fehlende

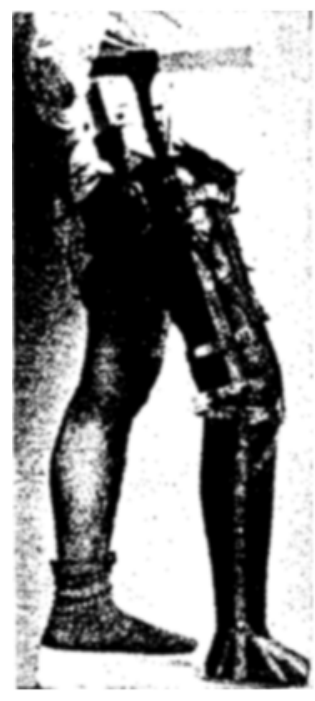

Fig. 13.

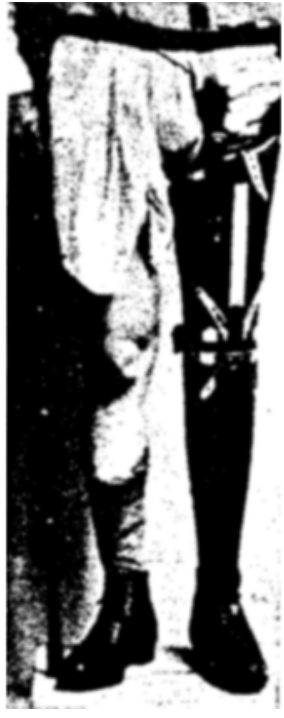

Fig. 14.

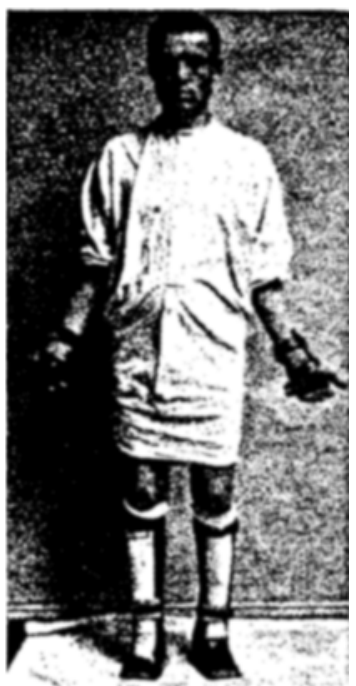

Fig. 15.

Stück am Fuss wird durch ein Stïck Kork ersetzt, das vorn in den Schuh hineingelegt wird, und nur den Zweck bat, dass der Schuh nicht einfällt.

Bei tragfähigen Unterschenkelstümpten, z. B. nach Operationen nach Symes oder Pirog off, werden einfache Unterschenkelapparate angefertigt, die unten mit einem Holzfuss versehen sind. Dieser hat auf der oberen Fläche eine Vertiefung, in welche der Stumpf hineinpasst. Der Apparat selbst wird durch einen einfachen Riemen oberhalb des Kniegelenkes festgebalten (Fig 15.) Auf dem Bilde fehlt dieser Riemen, da die Apparate nur in Roharbeit dargestellt sind.

Bei nicht tragfähigen Unterschenkelstümpfen wird eine trichterförmige Hülse aus Filz eingefügt, die den Stumpf trägt, ohne dass er mit seinem unteren Ende aufstösst (Kig. 16).

1) Über die Konstruktion der Gelenke vergl. Ho eftma a: Langeabecks Archiv Band 49 Heft 3. - Derselbe: Archiv für Klinische Chirurgie Band 69. - Derselbe: Verhandlungen der Deutschen Gesellschaft für orthopädiscbe Chirurgie Band 4.

Arch. 1. Orihop., Jechanoth. U. Unf.-Chir. XIYI, I. 


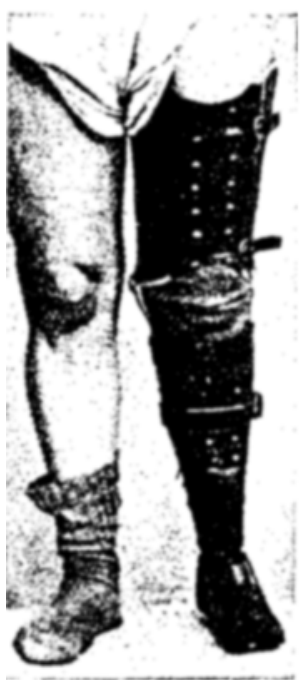

Fig. 16.

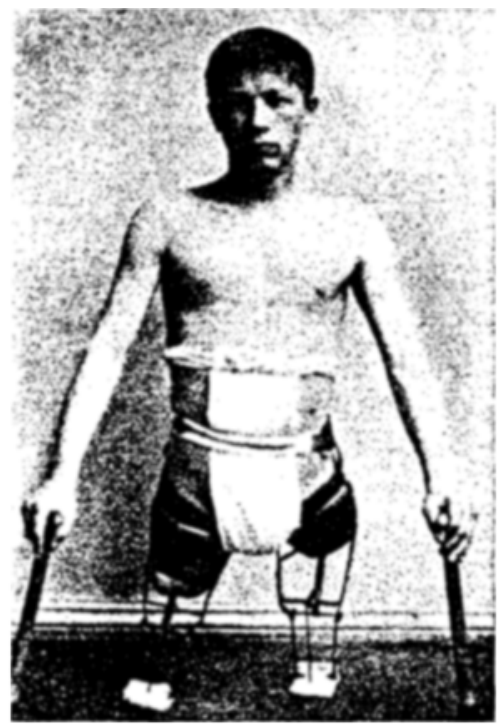

Fig. 1 .

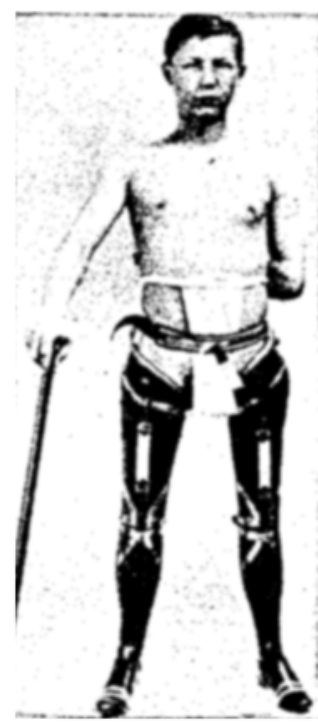

Fig. 18.

Wenn es sich darum handelt, Protbesen fiir solche Patienten anzufertigen, die beide Beine oberhalb der Kniegelenke verloren haben, kann man nicht die Apparate gleich von Anfang an in der natürlichen Länge herstellen ${ }^{1}$ ). Der Patient würde nämlich, aus Angst vor dem Hinfallen, nicht lemen, damit sicher zu gehen. Man muss daher in

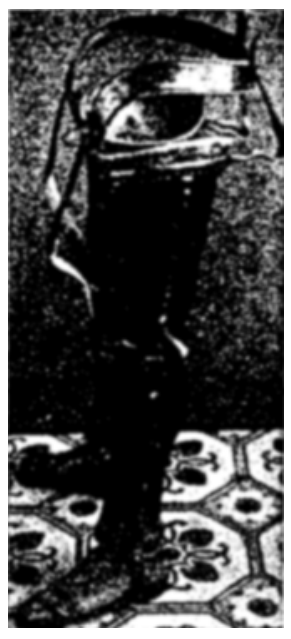

Fig. 19. solchen Fiallen zuerst Apparate mit ganz kurzen Beinen herstellen, und erst allmählich dieselben verlängern, wenn der Betreffende die nötige Sicherheit erlangt hat (Fig. 17 u. 18.)

Diese provisorischen Prothesen werden in einfacher Weise aus mit Scbellack getränkten Filzhülsen hergestellt und besitzen an den Seiten übereinander verschiebbare Schienen, so dass sie allmählich verlängert werden können. Erst wenn die normale Länge erreicht ist, werden die endgültigen Prothesen angefertigt.

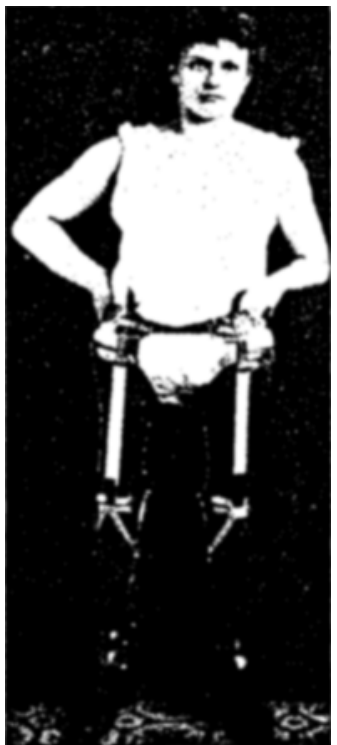

Fig. 20.

Es wäre noch zuletzt ein Apparat zu erwähnen, den Herr Prof. Hoeftman bei einem angeborenen Defekt

1) Vergl. Hoeft man: Vierteljahrsschr. f. gerichtliche Med. und äffentl. Sanitatswesen 3. Folge XLI. Suppl. - H. 
beider Beine konstruiert hat. Der Apparat besteht aus zwei künstlichen Beinen, mit exzentrisch nach hinten verlegten Knie- und mit beweglichen Fussgelenken. (Damals wurden die Fussgelenke noch beweglich gemacht.) Fig. 19.

Die Beinhülsen sind nicht wie sonst nach obon offen, sondern mit Leder geschlossen und schalenartig vertieft, so dass die beiden Beckenhälften sich darauf stützen könmen.

Die Prothesen sind durch einen Beckengurt verbunden. Derselbe wird durch eine Stahlschiene verstärkt, und hat den Zweck, dass die Prothesen in der richtigen Lage am Körper festgehalten werden (Fig. 20). Die Verbindung zwischen dem Gurt und den künstlichen Beinen ist nicht seitlich, sondern an der Vorderseite durch ein Scharnier bergestellt. Wenn also der Patient durch Seigen des Körpers nach der einen Seite, das Köpergewicht auf das eine Bein

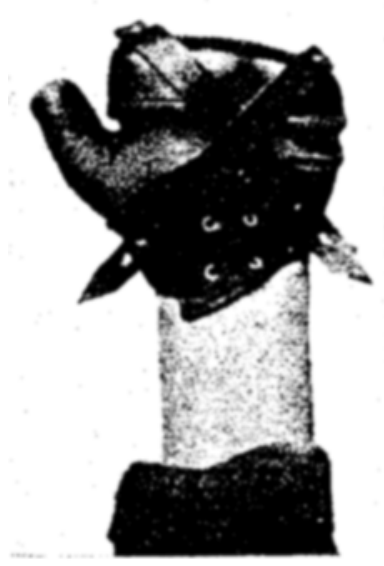

Fig. 21.

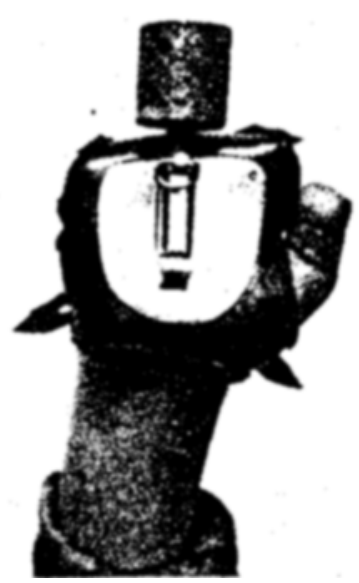

Fiz. 22.

verlegt, pendelt das andere von selbst um Schrittlänge nach vorne. Durch Neigen des Körpers nach dieser Seite, wird dieses Bein nun festgestellt und jenes Bein pendelt nach vorne, wodurch das Gehen möglich wird ${ }^{2}$.

Wir kommen nun zu den Prothesen für die oberenExtremitäten. Das durchgehende Prinzip bei denselben ist, dass sie möglichst einfach lonstruiert sind, so dass sie stabil und widerstandsfähig werden und wenig Reparaturen erfordern.

In solchen Fällen, in denen die Hand noch so weit erhalten ist, dass die Prothese um das Handgelenk befestigt werden kann, werden die Apparate so konstruiert, wie Fig. 21 und 22 zeigen. Sie sind an der Streckseite zum Schnüren eingerichtet und bekommen an der Hohlhandseite eine Metallplatte mit einer Düse zum Einstecken der verschiedenen Ansatzstücke.

Auf dem Bilde (Fig. 22) ist ein Ansatzstück eingesetzt, das zum Halten eines Spatenstiels berechnet ist. Mit anderen Ansätzen können die Patienten staken, mähen, Gewichte heben, nähen, stricken, schreiben usw.

I) Cber nähere Details vergl. Hoeftman, Langenbecks Archiy Band 39, Heft 3. 
Falls der Daumen vollständig erhalten und beweglich ist, wird an der Prothese, an geeigneter Stelle, ein aus Leder hergestellter pilzförmiger Knopf angebracht, gegen welchen der Daumen opponiert werden kann, so dass der

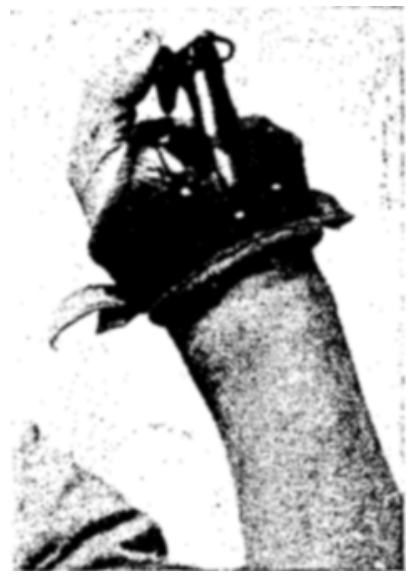

Fig. 23. Verletzte auch ohne andere Hilfsmittel damit Gegenstände fassen kann. (Fig. 23).

İhnliche Apparate können auch angefertigt werden, wenn die Finger erhalten, aber in Streckstellung versteift sind.

Wenn die Hand gänzlich fehit, ist das Aussehen des Apparates wesentlich anders. Die Konstruktion richtet sich auch nach der Länge des Unterarmstumpfes. Ist derselbe lang genng, d. h. mebr als $13 \mathrm{~cm}$, wird die Prothese so angefertigt, wie Fig. 24 zeigt. Sie besteht aus einer an der Beugeseite verschnürbaren Hülse, an deren Ende die Düse angebracht ist. Am oberen Ende der Unterarmbülse sitzen 4 Riemen, die mit einer Manschette verbunden sind, welche oberhalb des Ellenbogengelenkes angelegt wird, um zu verhüten, dass der Apparat rom Arm heruntergleitet.

Ist der Unterarmstumpf kürzer als $13 \mathrm{~cm}$, kann eine Konstruktion, wie sie eben beschrieben, nicht gebraucht werden. Der Stumpf würde sonst aus der Unterarm-

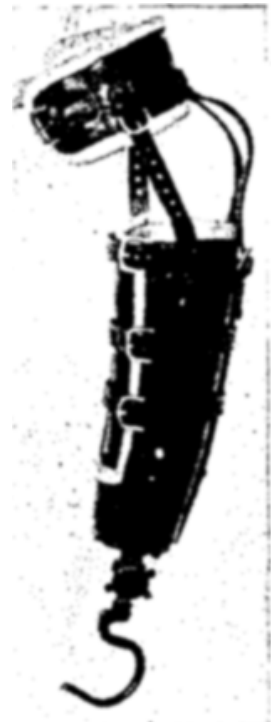

Fig. 24. hülse herausgleiten. Es muss daher in solchen Fällen auch für den Oberarm eine feste Hülse angefertigt werden, die mit dem Unterarmteil durch ein Scharnier verbunden wird. Das

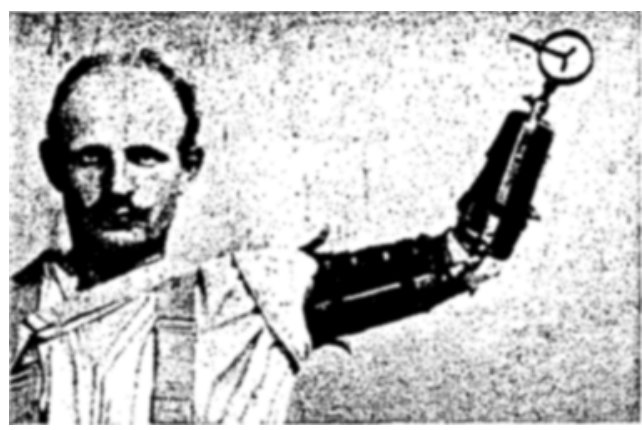

Fig. 25. Gelenk darf aber nicht die volle Streckung des Ellenbogens gestatten, damit der Stumpf nicht herausgleitet (Fig. 25).

Hat die Amputation oberhalb des Ellenbogens stattgefunden, besteht die Prothese aus einer Oberarmhülse, die durch Schulterriemen an der anderen Körperseite befestigt wird. Das Ellenbogengelenk ist aus einer mit Löchern versehenen Scheibe gebildet, in welche ein am Unterarmteil befindlicher Zapfen eingeschneppt werden kann. Hierdurch kann das Gelenk in jeder beliebigen Winkelstellung festgestellt werden ${ }^{1}$ ). Das

1) Vergl. Ho eftman: Verhandl. d. Deutsch. Gesellsch. für Orthopädische Chir. Bd. 6 u. 11. 
unterste Ende des Unterarmteils trägt die übliche I)üse (Figuren 26 und 27 ).

Wenn beide Oberarme amputiert sind, ist es schwierig, eine wirkliche

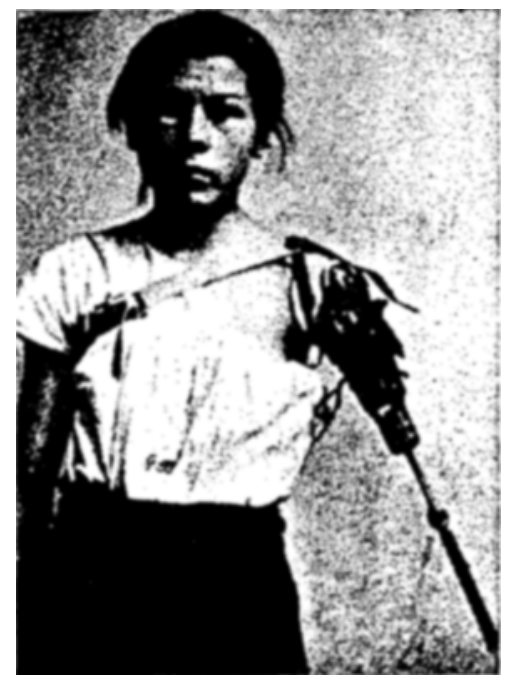

? Fig. 26. Arbeitsprothese herzustellen. Es gelingt jedoch, wenn die Oberarmstümpfe nicht gar zu kurz sind, auch solche Patienten so weit $z \mathbf{u}$ bringen, dass sie bei ihren leiblichen Bedürfnissen, Essen, Trinken etc. sich allein behelfen können. Es ist auch verhältnis. mässig leicht, ilınen das Schreiben beizubringen, wenn der Stumpf nur $10 \mathrm{~cm}$ lang ist.

Ein näheres Ein. gehen auf die immerhin sehr komplizierte

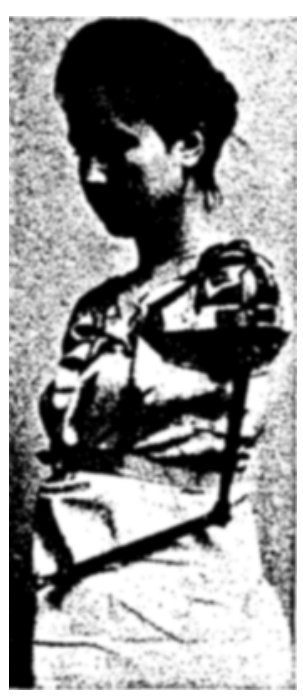

Fig. 27.

Konstruktion derartiger Apparate muss hier aus eingangs erwähnten Gründen unterbleiben. Für diejenigen, die sich etwa hierfür interessieren, wird aut die Inaugural-Dissertation ron $\boldsymbol{H}$ eis s le $\mathrm{r}^{1}$ ) uber einen derartigen in hiesiger Anstalt beobachteteu Fall hingewiesen (Fig. 25).

\section{Fehlerhafte Apparate.}

Es ist interessant zu sehen, wie die Patienten manchmal durch selbst angefertigte Prothesen den Verlust eines Gliedes auszugleichen versuchen. Es sind mebrere solche Fälle hier beobachtet (Fig. $29-33)$, und zwar handelte es sich dabei stets um Unterschenkelamputationen. Die Patienten gingen alle mit gebengtem Knie, auch wenn das Kniegelenk vollkommen gestreckt werden konnte, was durch die Einfachheit der Prothesen erkiärt wurde. Um den Druck zu vermeiden, benutzten sie mitunter ganz primitive Polsterungen (Fig. 29-30).

Einzelne von diesen Prothesen waren, wenn auch grob gearbeitet, so doch recht zweckmässig. Andere erfüllten nur mangelbaft ihren $Z_{\text {weck. So }}$

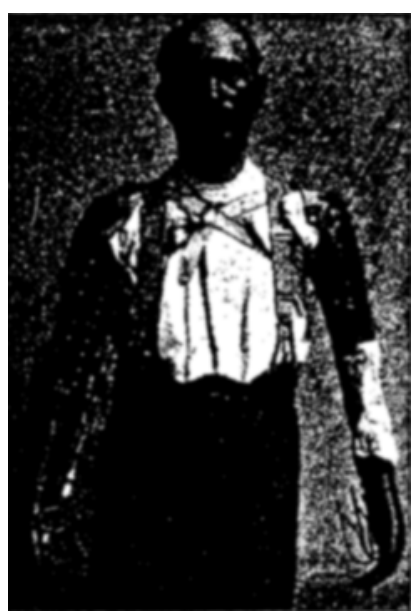

Fig. 28. war die Prothese (Fig 30) nach oben zu kurz und batte hier nor einen einfichen schmalen liemen, dex um das Bein oberialb des Knies angeschnalit

') Heissler, s. dieses Arcbiv Bd. XII, Heft 4, 1913. 
wurde, ohne genïgende Stütze zu geben. Man sieht auf dem Bilde (Fig. 31), wie das Bein durch den Riemen allmählich eingeschnüt worden war.

Als Pendant zu diesen selbst konstruierten Apparaten mag die Abbildung einer Prothese beigefügt werden, die von einem Bandagisten angefertigt

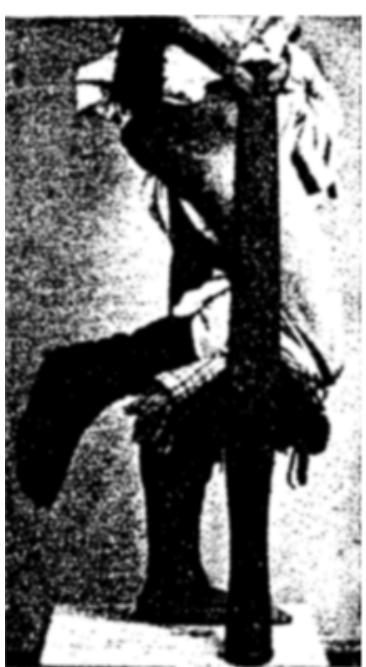

Fig. 29.

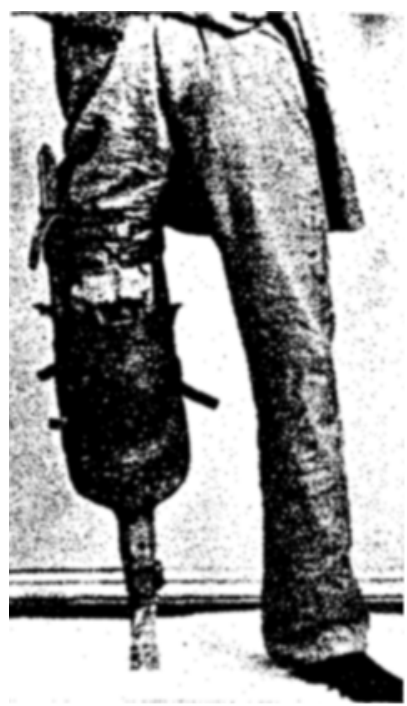

Fig. 32.

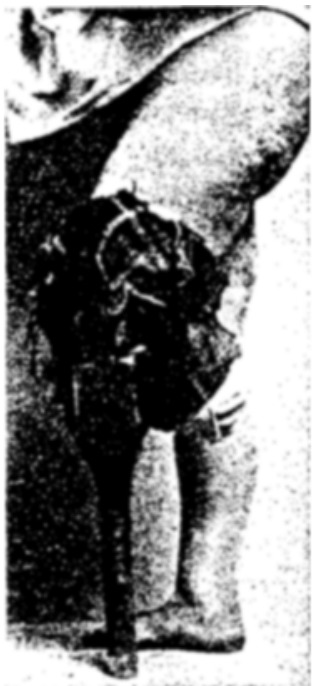

Fig. 30.

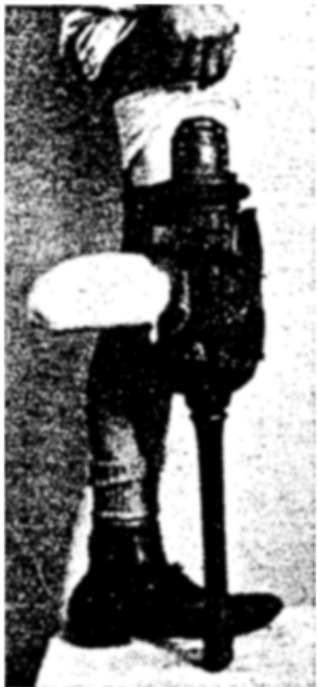

Fig. 33 .

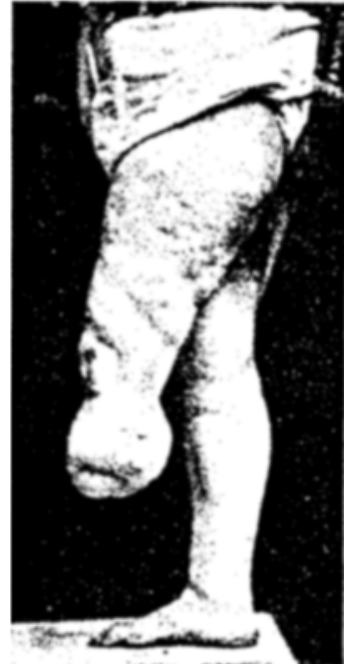

Fig.:31.

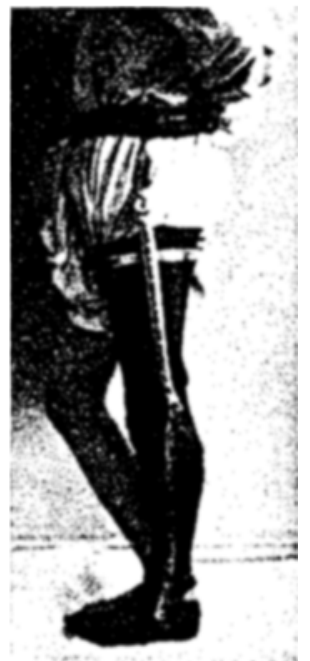

Fig. 34.

war und ein Beispiel dafür ist, wie derartige Prothesen nicht angefertigt werden sollten. Es genügt ja ein Blick auf das Bild, um zu erkennen, dass die Prothese viel zu kurz ist, so dass der Patient nur mit gebogenem rechten Bein stehen konnte und folglich beim Gehen stark hinken musste (Fig. 34). 
Auch bei der Anfertigung von Korsetts wird manchmal gesündigt. Fig. 35 zeigt einen Mann, der einen Bruch des zweiten Brustwirbels erlitten hatte und deswegen ein Korsett bekam, das von einem Bandagisten angefertigt war. Der Sitz der Verletzung ist auf dem Bilde durch Anzeichnen mit Tusche kenntlich gemacht. Man sieht, dass dieverletzte Stelle höher liegtals das Korsett hinaufreicht.

In Fig. 36 und 37 handelte es sich um einen Bruch des 8. Brustwirbels. Auch in diesem Falle ist das Korsett viel zu niedrig. Es war rorne aufgeschnitten, hatte aber keine Schnürrorrichtung, sondern wurde, nie das Bild zeigt, durch eine einfache Binde zusanmengehalten. Es ist selbstverständlich, dass solcbe Korsetts eine Stütze für die erkrankten Wirbel absolut nicht abgeben, und also den beabsichtigten $\mathrm{Zweck}$ keineswegs erfüllen.

\section{Stïtzapparate.}

In diesem Zusammenhang wäre noch etwas über die Apparate, wie sie hier als

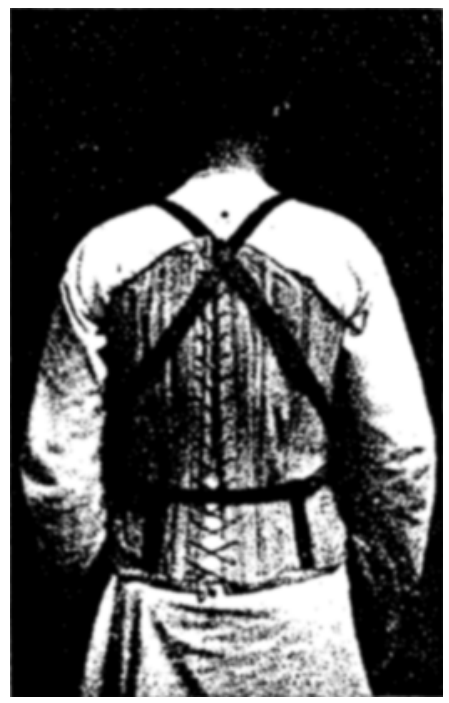

Fig. 35 .

Stütze bei verschiedenen Erkrankungen angefertigt werden, zu erwähnen. Dieselben lassen sich in grössere Gruppen einteilen:

1. Apparate für die Gelenke,

2. $\quad$ bei Lähmungen,

3. $\quad$ " nicht verheilten Frakturen,

4. " "Platt- Kilump- und Spitzfiissen.

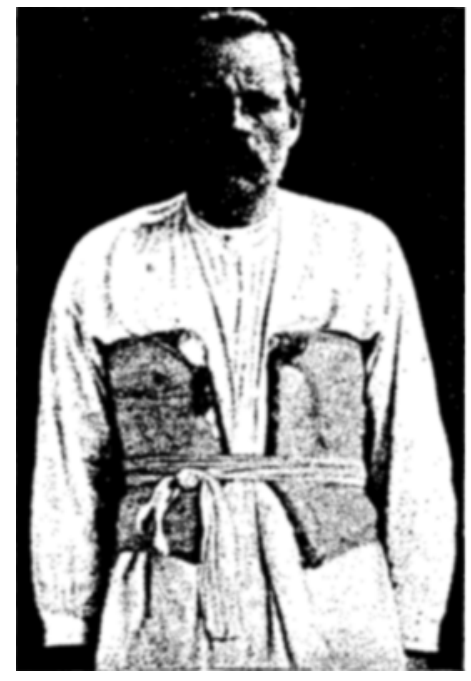

Fig. 36 .

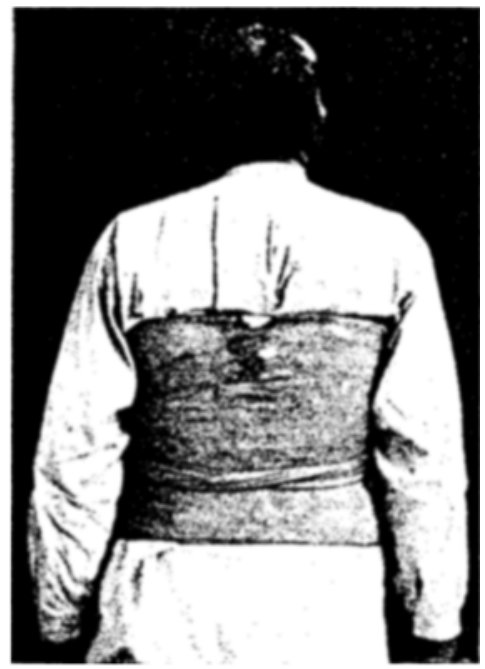

Fig. 37. 


\section{Apparate für die Gelenke.}

Linter den Apparaten für die Gelenke kommt zunächst der von Herrn Professor Hoeftman angegebene Coxitisapparat in Betracht. Derselbe hat

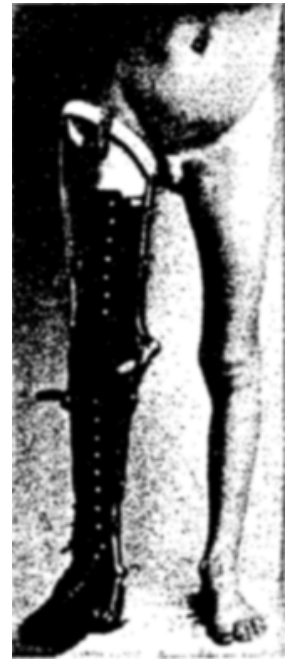

Fig. 38. den Zweck, das Hüftgelenk bei jeder Stellung des Beines zu entlasten. Er besteht in der Hauptsache aus je einer Hülse fiir hen Ober- und Unterschenikel mit doppelten Seitenschienen rerbunden. Das eine Schienenpaar geht bis zum Becken hinauf und trägt hier einen Reitring, der sich gegen das Beclien stützt. Nach unten setzt sich dasselbe Schienenpair in den Fussteil fort, der aus einer Stahlsoble und Lederkappen mit Schnürvorrichtung besteht, um den Fuss zu fixieren. Da ferner die Achse für das Kniegelenk am Apparat um $21 / 2-3 \mathrm{~cm}$ nach hinten verlegt ist, wird erreicht, dass das Bein auch in gebeugter Stellung in Extension gehalten wird ${ }^{\mathbf{1}}$. Fig. 38 น. 39.

Für Unbemittelte hat Herr Professor

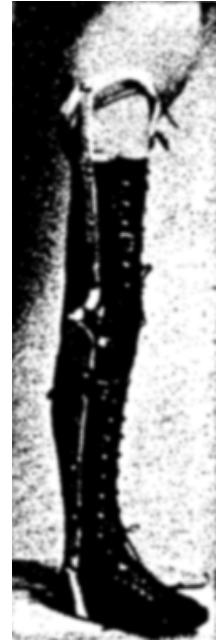

Fig. 39.

Hoeftman einen sebr einfachen Apparat zur Entlastung des Hüftgelenks konstruiert. Derselbe besteht aus einem U-förmig gebogenen Bandeisen, das durch Wasserglashanfstreifen verstärkt wird. Am oberen Teil wirl er

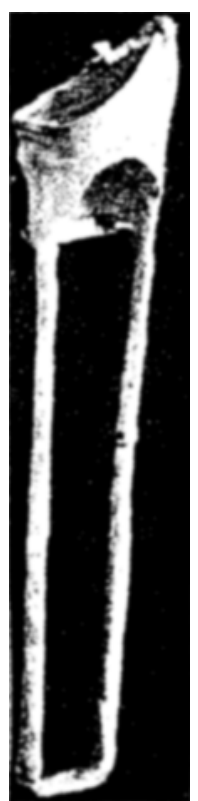

Fig. $40 \mathrm{a}$. durch Gazebinden ringförmig gearbeitet. In der obersten Kante dieses Ringes ist ein Gummischlauch eingearbeitet, um als Sitzring zu diener. Der Apparat reicht bis zum Becken hinituf und wird ein wenig länger gemacht ais das kranke Bein. Der Fuss desselben wird am unteren Ende des Apparates durch Heftpflasterstreifen befestigt. Hierdurch wird erreicht, dass das

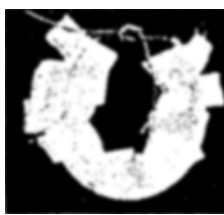

Fig. $40 \mathrm{~b}$. Bein auch in Sitzen in Extension gehalten wird ${ }^{2}$ ). (Fig. 40).

Dieser Apparat kann auch zur Entlastung des Kniegelenkes verwandt werden. Sonst werden die Apparate für diesen $L$ weck ähnlich kunstruiert wie die vorher beschriebenen Prothesen bei Oberschenkel-Amputationen. Das Kniegelenk kann dabei entweder steif oder beweglich gemacht werden.

Zur Fntlastung des Fussgelenkes wird ein ähnlicher Apparat angefertigt, nur reicht die Oberschenkelhülse nicht bis zum

1) Naheres über den Apparat siehe die Inaugural-Dissertation von Hildebrandt 1891.

") Eoeftman: Einige Mitteilungen ans der Praxis. Archiv für klin. Chir., Bd. 69. 
Bericht aber die Privatklinik des Herra Professor Dr. Hoeftman in Königsberg Pr. 25

Becken hinauf, and das liussgelenk ist steif und ausserdem noch mit Streben verstärkt.

Bei habituellen Luxationen im Schultergelenk bekommt der Patient einen Apparat, der aus einer Schulterkappe und einer Oberarmbiilse besteht. Ent-

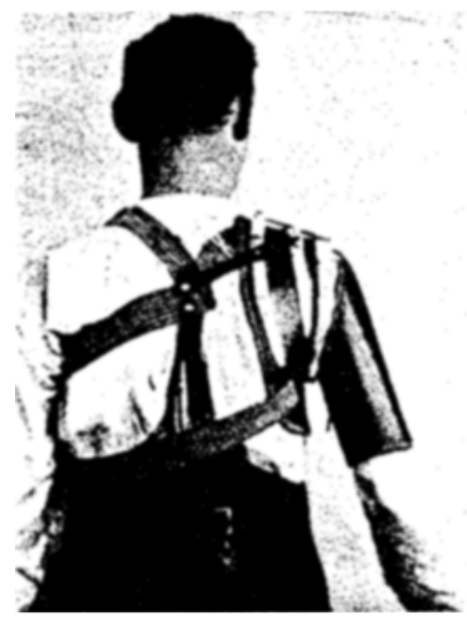

Fig. 41.

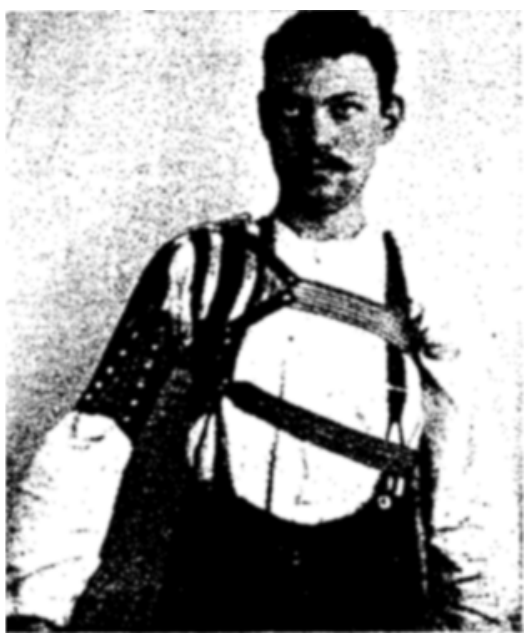

Fig. 42.

weder sind diese beiden durch ein Gelenk verbunden, das zwar Drehbewegungen erlaubt, aber das Heben des Armes über eine gewisse Höhe verhindert (Fig. 41 u. 42), oder aber die Armhülse wird durch Riemen mit der Schulterkappe verbunden, wodurch ebenfalls das zu hohe Heben des Armes verhütet wird. (Fig. +3).

II. Apparate bei Lähmungen.

Fs kommen hier in der Hatuptsache nur Lähmungen der unteren Extremitäten in Iirage. Bei Peroneuslähmungen bestehen die Apparate aus einem Fussteil und einer Unterschenkelhülse, die beweglich mit einander verbunden sind. Das Heben der Fussspitze wird durch an der Streckseite des Fussgelenkes lireuzweise angebrachte Gummizüge bewerkstelligt.

Bei Lähmungen des ganzen Beines kommt noch eine Oberschenkelhülse hinzu, die mit der Unterschenkelhülse durch ein Gelenk verbunden ist. An der Vorderseite des Kniegelenkes befindet sich eine Pelotte, die durch

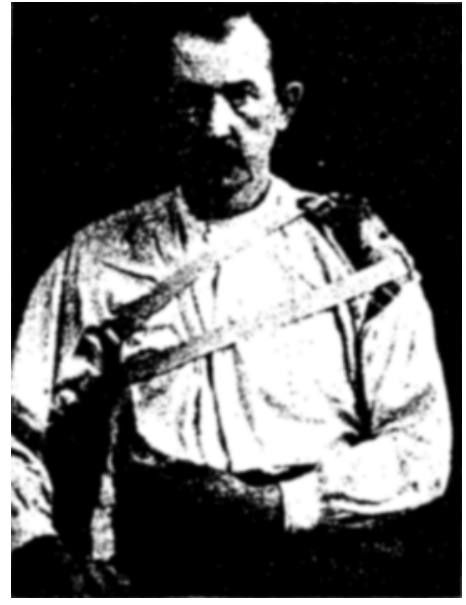

Fig. 43. Gummizug an der Ober- und Unterschenkelhülse befestigt ist; oder es wird eine Rapierklinge in Verbindung mit Gummizügen verwendet, ev. in Kombination mit exzentrischen Gelenken, die aber hier nicht zu weit hinter 
das Kniegelenk verlegt werden dürfen, weil sonst Distorsionen des Kniegelenkes rorkommen.

In diesem Zusammenhang wïren noch besonders die sogenannten "Handgänger zu erwähnen. Es handelt sich um Fälle von spinaler Kinderlähınung, in welchen beide Beine total gelähmt sind. Einige solcher Patienten gewöhnen

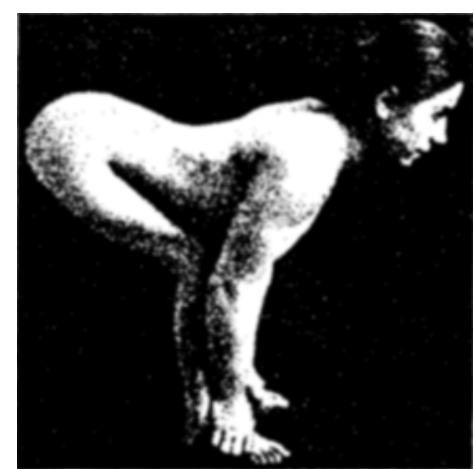

Fig. 44.

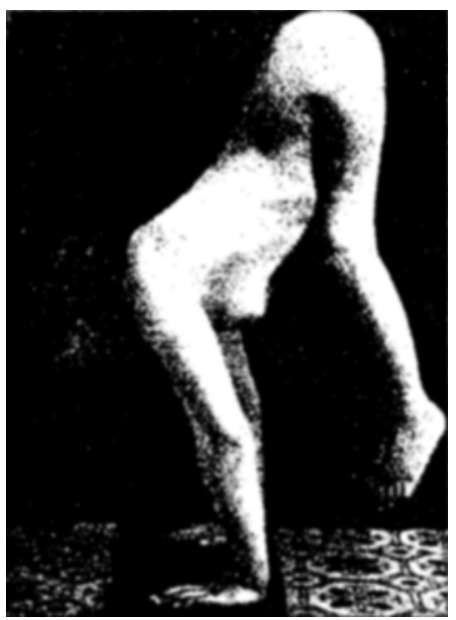

Fig. 45. sich einen eigenartigen Gang an, indem sie mit Hilfe der Hände die Füsse vorwärts setzen. Sie können sich auf diese Weise recht schnell, sogar im Laufschritt, vorwärts bewegen. Der Körper nimmt dabei eine horizontale Stellung ein. Die Beine sind in den Hüftgelenken extrem gebeugt, so dass die Oberschenkel vollständig dem Rumpfe anliegen, während die Unterschenkel ungefälir in der Witte des Körpers senkrecht stehen (Fig. 44).

Andere haben sich sogar gewöhnt, direkt auf den Händen zu gehen, wobei der Pumpf hochgehalten wird und die Beine schlaff herunterbängen (Fig. 45.)

Es sind hier fünf derartige Fälle beobachtet und zum Teil schon beschrieben worden 1 .

Was die Behandlung derartiger Fälle anlangt, so handelt es sich zunächst darum, die Kontraktur in den Hüftgelenken und die hierdurch bedingte starke Lordose $z \mathrm{u}$ beseitigen. Der Patient wird so hingelegt, dass die Wirbelsäule der Unterlage flach aufliegt. Die Beine stehen dann meistens in einem Winkel von vielleicht $60^{\circ}$ nach oben and werden anfangs in dieser Stellung extendiert. Ist die Lordose beseitigt, geht man zu der Beseitigung der Hüftgelenks-Kontrakturen über. Dazu bekommt der Patient zunächst ein Gipskorsett, wodurch der Rumpf vollständig steif gehalten wird. Das Korsett wird in sitzender und nach rorne gebeugter Stellung angefertigt, um somit jede Lordose zu vermeiden. Dann werden an die Beine Gipshanlschienen angelegt, die zwecks Extension mit Ringen verseben sind. Die Extension - 15 bis 20 Pfund an jedem Bein - geschieht zunächst in einem Winkel von ungefähr $45^{\circ}$. Würde man gleich horizontal exten-

1) Hoeftman: Einige Witteilungen aus der Praxis, Archiv für klinische Chirargie Bd. 69. (Festschrift für ron $\mathrm{E} s \mathrm{march}$ ). 
Bericht über die Priratklinik des Herrn Professor Vr. Hoeftman in Königsberg Pr. 27

dieren, liefe man Gefahr, die Hüftgelenke zu luxieren. Daher werden die Beine erst allmählich gesenkt, bis sie zur Horizontalen gelangt sind.

Ist dies erreicht, wird der Rumpf mittelst eines Korsetts in gerader Haltung fixiert und die Beine werden mit den anfangs boi den Lähmungen erwähnten Apparaten versehen.

Zunächst müssen die Patienten an Gehstühlen und dann an Kruicken Gehen lernen. Zuletzt bewegen sie sich je nach ihrer Geschicklichkeit mit 1 bis 2 Stöcken (Fig. 46) ${ }^{1}$ ).

III. Apparate bei nicht verheilten Frakturen.

Die bei Pseudarthrosen angefertigten Apparate sind denjenigen ähnlich, die bei Gelenkerkrankungen erwähnt sind. Sie brauchen daher hier weiter keine Erläuterung. Nur muss bei ihrer Anfertigung darauf geachtet werden, dass sie lang genug sind, so dass die Pseudarthrose auch tatsächlich gestïtzt wird. Pseudartbrosen der unteren Extremitaten ptlegen meist immer dabei zu heilen, falls nicht die Dislokation eine zu grobe war. Es kommen selbstverständlich auch Fälle vor, bei denen nur durch eine Operation eine Heilung zu erzielen ist, wenn $\%$. B., wie hier zweinal beobachtet wurde, die Tibia mit der Fibula zusammengewachsen war.

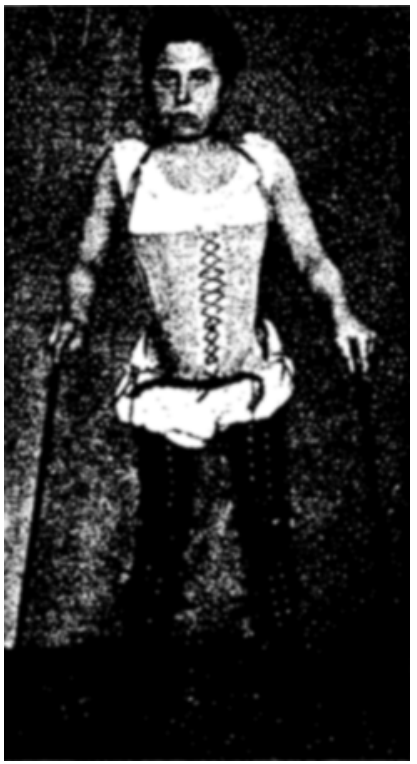

Fig. 46.

Es ist hier sogar bei veralteten Schenkelhalsbrüchen, die erst $1 / 2-1$ Jahr nach der Verletzung in Behandlung kamen, in mebreren Fällen gelungen, durch zweckmässige Apparate eine Heilung zu erzielen.

IV. Apparate bei fehlerhafter Fusstellung.

Bei leichteren Fällen von Finick- und Plattfuss kommt man mit einer einfachen Stützsohle aus. F.s wird zunächst ein Gipsmodell von dem betreffenden Fuss angefertigt, und darauf die Sohle entwerler aus Stahl oder ans Kork gearbeitet. Die Stahlsohlen werden mit Leder bezogen und nachdem der Patient sie zur Probe getragen bat, noch verzinnt oder vernickelt, um das Rosten zu verhüten.

Die Korksohlen werden durch Einlagen von dünnen Stahlschienen verstärkt und mit Leinen überzogen. Sie sind ja nicht so dauerhaft wie die Stahlsohlen, haben aber dafïr den Vorzug grosser Leichtigkeit. Anch können sie, wenn einzelne Teile am I uss druckempfindlich sind, an entsprechenden Stellen ausgehöhlt und durch Schwammgummi ersetzt werden ${ }^{2}$ ). Bd. 2.

1) Hoeftman: Verhandlangen der Dentschen Gesellachaft für orthopadischeChirargie

3) Hoeftman: Zeitschrift für orthopidische Chirurgie, Bd. 25. 
In schwereren Fällen von Knick- und Plattfüssen muss vorher ein Redressement des Fusses stattfinden. Dasselbe wird entweder allmählich durch einen Gummizug oder, wenn dies nicht ausreicht, durch ein Redressement in Narkose ausgeführt ${ }^{1}$ ).

Da bäufig doch zur Nachbehandlung Apparate getragen werden müssen, so wird in diesen Fällen so vorgegangen, dass gleich bei der Aufnahme ein Modell von dem Fuss in der fehlerhaften Stellung angefertigt wird, das dann in geeigneter Ebene durchschnitten und in gut korrigierter Stellung wieder zusammengeleimt wird. Es kann dann sofort mit der Herstellung des Apparates begonnen werden, so dass nach dem Redressement des Fusses der Apparat schon fertig gearbeitet ist. Es kürzt dies die Behandlungsdauer nicht unerheblich ab.

In ähnlicher Weise wird der Klumpfuss behandelt. Die Apparate bestehen aus einer Cinterschenkelhülse mit Fussteil, wodurch der Fuss in der gewünschten Lage festgebalten wird. Darüber kommt ein besonders gearbeiteter Schuh.

Auch bei Spitzfüssen kommen Gummizüge zur Verwendung, um die fehlerhafte Stellung zu beseitigen. Ist das Fussgelenk jedoch ankylotisch, wird eine Korkeinlage angefertigt, die vorne niedrig, hinten aber so hoch ist, dass die fehlerhafte Stellung ausgeglichen wird.

\section{Skoliosen-Behandlung.}

Über die hier verwandten Behandlungsmethoden bei Skoliosen hat schon Strube in seinem Bericht über die Klinik so ausführlich berichtet, dass ein näheres Eingehen auf dieses Thema sich erübrigt ${ }^{1}{ }^{2},{ }^{2}$. Es genügt daher eine kurze Übersicht.

Zunächst werden die Skoliosen an dem Zander'schen Messapparat gemessen, und diese Messung wiederholt sich etwa alle 4 bis 5 Wochen. Hierdurch gewinut man einen objektiren Beweis für die eingetretenen Veränderungen, Im ferneren Verlauf der Behandlung müssen die Patienten im Zander-Institut Übungen vornebmen, ausserdem liegen sie auf Streckbetten. Diejenigen, die in der Klinik stationär behandelt werden, liegen bis zu 6 Stunden und üben zweimal täglich an den Zander-Apparaten, so dass sie so gut wie den ganzen Tag in Behandlung bleiben.

Ferner kommt Massage and in vereinzelten Fällen eine Futterkur zur Verwendung. Bei schwereren Fällen bekommen auch die Patienten Gips- oder Stoffkorsetts.

Sind die Skoliosen schon sebr starr, wird ein Redressement in Narkose vorgenommen. Die Armo und Beine von dem Betreffenden werden mit feuchten Leinenbinden umwickelt und an den Händen und Füssen eine Schlinge gemacht, an der der Patient nachher mit dem Gesicht nach anten in hori-

1) Strube: Bericht aber die Hoeftman'sche Klinik. Zeitschrift für orthopadische Chirurgie, Bd. 9.

3) Vgl. auch Hoeftman, Zeitsehrift für orthopädische Chirurgie, Bd. 9. 
zontaler Stellung aufgehängt wird. Durch einen Flaschenzug kann eine starke Extension ausgeiührt werden, während welcher die Skoliose und vor allem der Rückenbuckel durch Handkralt möglichst redressiert wird. Noch während der Patient in Extension sich befindet, werden Gipsbinden umgewickelt, und erst nachdem das Korsett steif geworden ist, wird mit der Extension nachgelassen. Durch dieses Verfahren ist manchmal eine Verlängerung der Körpergrösse um $6 \mathrm{~cm}$ erreicht. Das Korsett muss, wenn es irgend geht, 14 Tage liegen bleiben. Nur wenn die Kinder es nicht so lange aushalten, wird das Korsett früher aufgeschnitten. Nach Abnahme des ersten Verbandes bekommen die Patienten ein abnehmbares Gipskorsett, damit sie an den Zander-Apparaten Übungen vornehmen können. Die Gipskorsetts werden ev. alle 8 Tage erneuert und jedesmal wird versucht, ein weiteres Redressement zu erzielen.

Die Stoffkorsetts sind für längeres Tragen berechnet, sie werden häufig so angefertigt, dass durch dieselben ein Redressement ausgeübt wird. Manchmal aber dienen sie nur kosmetischen $\mathrm{Z}$ wecken.

\section{Spondylitisbehandlung.}

Das bier bei der Behandlung von Spondylitis durchgehende Prinzip heisst die Patienten so kurz wie wöglich zu Bett liegen zu lassen. Nur in schweren Fällen mit Läbmungen erfolgt daher die Behandlung durch Gipsbetten, sonst durch Korsetts.

bei Erwachsenen werden Filzkorsetts bevorzugt, weil sie dauerbafter sincl. Bei Kindern werden dagegen Gipskorsetts angelegt, da sie infolge des

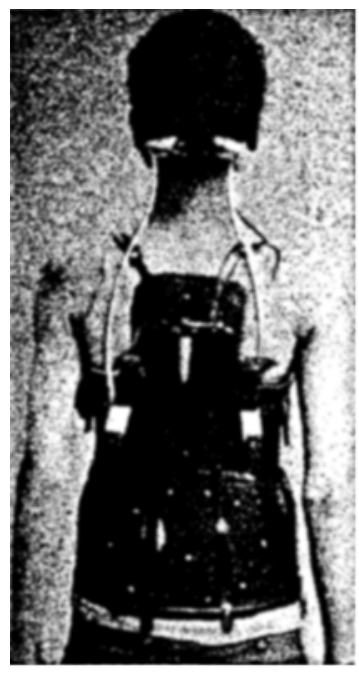

Fig. 47 .

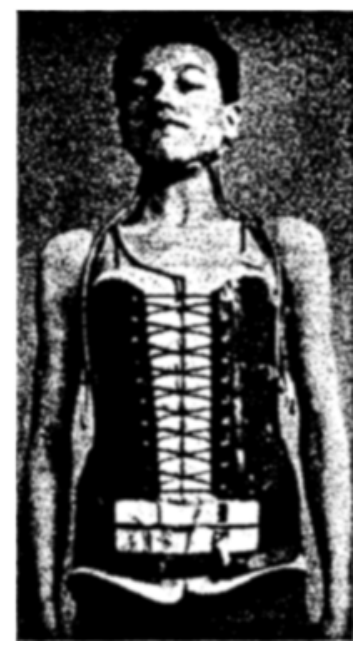

Fig. 48.

Wachstums öfter gewechselt werden müssen. Bei hohen Spondylitiden werden die Gipskorsetts entweder mit einem Kopfteil versehen, so dass nur die Ohren und das Gesicht frei bleiben, oder es wird ein Halsring mit Stützen (Fig. 47 und 48) 
oder ein Jurimast (Fig. 49) angebracht. Erwachsene erhalten in solchen Fällen eine Kopfstütze, die anf den Schultern ruht.

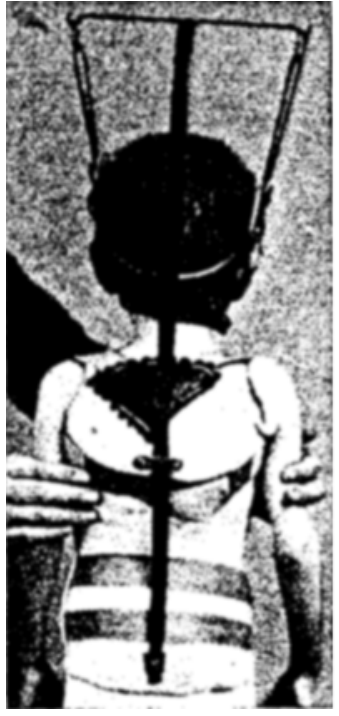

Fig. 49.

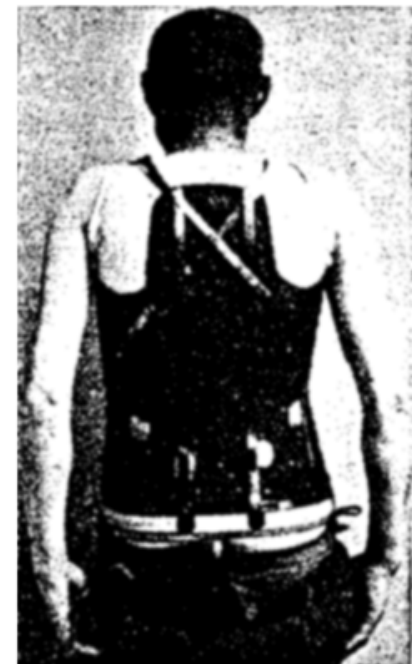

Fig. 50 .

Die Filzkorsetts (Fig. 50) werden auf Modellen genrbeitet und bestehen aus dickem Filz, der mit Schellack getränkt und durch Stahlschienen verstärkt wird. Sie werden vorne durch eine Gummischn'r und Schnallen geschlossen. Diese Korsetts haben sich sehr gut bewährt, da sie dauerhaft sind, ihre Her-

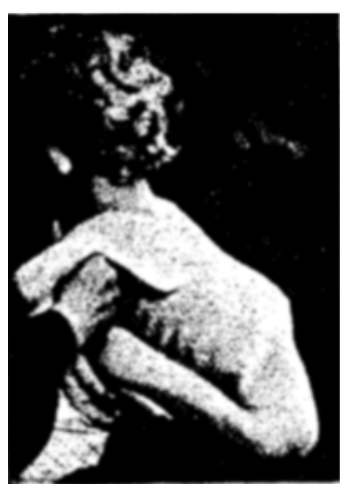

Fig. 51.

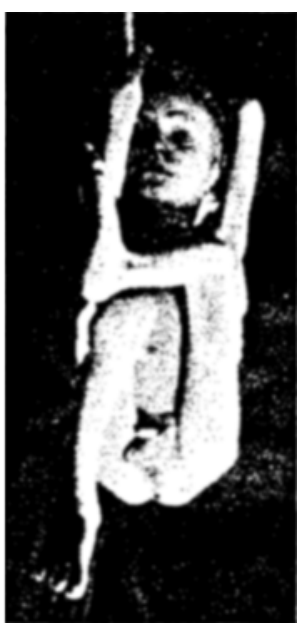

Fig. 52.

stellung nicht so schwierig wie z. B. Zelluloid-Korsetts ist, und Reparaturen leicht rorzunehmen sind. Ausserdem haben sie den Vorteil, dass man beliebige Stellen weich lassen kann. 
Berıcht iber die Privatklinik des Herrn Professor Dr. H o eftm a n in Königsberg Pr. 31.

Figg. 51 und 52 zeigen einen besonders schweren Fall ron Spondylitis, mit mehreren Fisteln und mit Kontrakturen in den Beinen. Das Kind wurde zunächst durch Gipsbett und Extension behandelt und belsam dann später Korsetts. Der Fall war anfangs ziemlich trostlos, das Resultat der Behandlung war aber sehr gut. Schon nach zwei Monaten konnte das Kind mit einem Gipskorsett gehen ${ }^{1}$ ).

Das Bild (Fig. 53) ist drei Jihre nach Beginn der Behandlung aufgenommen, und man sieht daraus, dass es dem Patienten gut geht.

Über die operative Behandlung der Spondylitis vergl. Strubes Bericht über die Klinik ${ }^{2}$ ).

\section{Kongenitale Juxationen.}

Über die Bebandlungsmethoden der kongenitalen Hüftgelenks-Luxationen wäre folgendes zu erwähnen.

Wenn es sich um Kinder handelt, wird stets eine unblutige Reposition in Narkose vorgenommen. Der Oherschenkel wird unter Rotation nach innen stark im Hüftgelenk gebengt. Darauf folıgt die Zirkum-

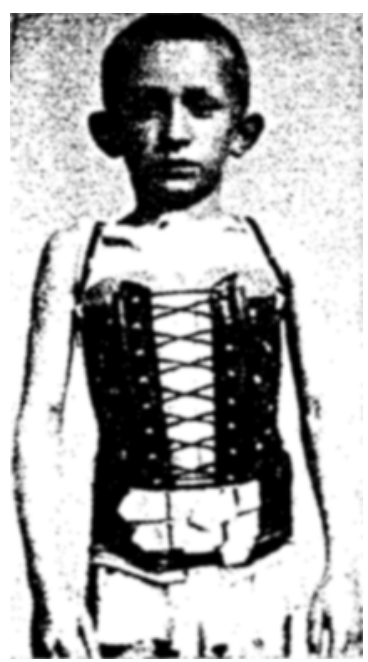

Fig. 33. duktion des Knies, verbunden mit Innenrotation des Oberschenkels, bis dieser etwa rechtwinklig vom Körper absteht. Das Bein wird dann in dieser Lage fixiert und zwar hat Herr Prof. Hoeftman hierfür einen Apparat konstruiert, der hier im Bilde beigefügt wird (Fig. 54).

Der eine Zeitlang gemachte Versuch, das Bein gleich nach der Reposition in Extensionsstellung nach unten $z u$ bringen und $z u$ fixieren, ist ganz aufgegeben, weil dabei beim geringsten Fehler eine Reluxation auftrat, so dass der Erfoly nur in seltenen Fällen ein dauernder war, wäbrend jetzt nur in ganz seltenen Fällen ein Misserfolg eintritt.

Handelt es sich um Erwachsene, so kann die Reposition selbstverständlich nur auf blutigem Wege erfolgen. Aber auch ohne Operation liann man aut andere Weise ein gutes Resultat erzielen. Im letzten Vierteljahr sind hier z. B. drei Patienten im Alter von 19,26 und 27 Jahren wegen einer kongenitalen Luxation behandelt worden. Sie batten aile angefangen, plötzlich schlechter zu gehen, und über Beschwerden zu klagen. Diese Verschlechterung war

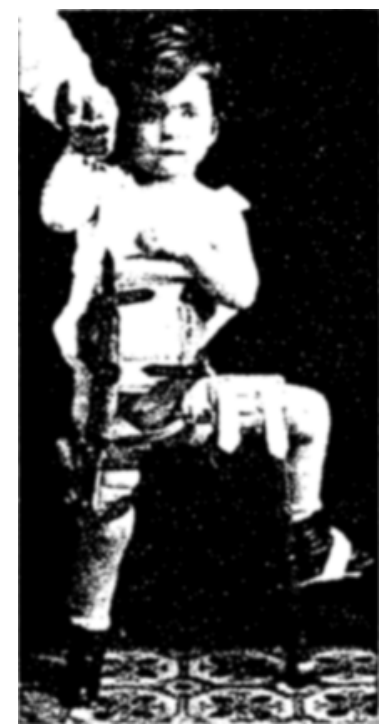

Fig. 54.

I) Hoeftman: Beiträge zur Gipsbettbehandlung der Wirbelsäulenerkrankungen. Verhandlang der deutschen Gesellschaft für orthopädische Chirurgie Bd, 10.

2) Strube: Zeitschrift für orthopadische Chirurgie Bd. 9. 
darauf zurückznführen, dass der Allgemeinzustand geschwächt war, wodurch die Muskeln der Hüftgegend insuffizient waren. In allen drei Fällen hat eine geeignete Nassage kombiniert mit Übungen an den Zander-Apparaten die Schmerzen beseitigt und den Gang wiederum sehr wesentlich gebessert, so dass das Hinken fast ganz verschwand. Die Methode ist für solche Fälle dringend zu empfehlen.

\section{Behandlung frischer Frakturen.}

Frische Frakturen der Wirbelsäule werden so behandelt wie eine Spondylitis. Hat der Bruch im Bereich der Hals-Wirbelsäule stattgefunden, bekommen die Patienten also eine Kopfstätze, liegt die Verletzung im Bereich der Brustoder Lenden-Wirbelsäule, kommen Gipsbetten oder Korsetts zur Verwendung.

Bei Arm- oder Beinbrüchen gilt als allgemeines Prinzip, dass zirkuläre Gipsrerbände so gut wie nie angelegt werden. Statt dessen kommen nur Schienenverbände zur Anwendung. Diese Schienen werden entweder aus Gipshinden oder aus Gips-Hanfstreifen nach Beely ${ }^{1}$ ) hergestellt.

Die ersteren können noch dadurch haltbarer gemacht werden, dass zwischen die Schichten in heissem Wasser aufgeweichte Schusterspäne hineingelegt werden. Diese Schienen sind sehr leicht und lassen sich elegant formen.

Die zweite Art, die Gips-Hanf-Schienen, werden aus Hanfstrei fen hergestellt. die in der gewïnschten Länge geschnitten und durch einen Gipsbrei durchgezogen werden.

Der überschüssige Brei wird durch Hindurchziehen des Bündels zwischen den Fingern entfernt, und die Streifen dann parallel der Längsachse des Gliedes dicht nebeneinaniler auf die blosse Haut gelegt. Nach dem Erstarren wird die Schiene mit Binden festgewickelt. Da sie nur den halben Umkreis des Gliedes umfasst, kann dasselbe in der gewünschten Stellung ganz unbeweglich gehalten werden, bis der Gips erstarrt ist, wodurch jegliche fehlerhafte Stellung (Rekurvation, Spitzfuss, Plattfuss etc.) leichter vermieden werden kann, als bei zirkulären Verbänden. Ferner enstehen keine ischämischen Lähmungen, da der Verband, wenn es nötig sein sollte, jeden Augenblick gelockert werden kann. Ausserdem erlauben die Schienenverbände eine stetige Kontrolle und Behandlung etwaiger Wunden. Schliesslich ist es bei dieser Behandlung möglich, den Verband täglich abzunehmen und eine Massage auszuführen.

Wenn erforderlich, können gleich bei der Herstellung der Schienen Eisenringe eingegipst werden, um die Extremität später aufzubängen.

Gips-Hanf-Schienen können auch mit Bügeln aus Eisendraht und Gummizügen versehen werden, was bei der Behandlung von Platt- und Klumpfüssen gute Dienste leistet ${ }^{2}$.

Bei Brüchen des Oberarmhalses mit Dislokation muss der Patient $2 u$ Bett liegen und bekommt einen Extensionsverband, der nach oben aussen Schienen.

1) Beoly: Zar Behandlung einfacher Frakturen der Extremitäten mit Gips.Hanf-

2) Vergleiche Strube. Bericht über die Hoeft man'sche Klinik. 
gerichtet wird. Die Lage ist allerdings unbequem, es gelingt aber auch in solchen Fällen, in denen die Dislokation nicht vollständig beseitigt werden kann, eine freie Beweglichkeit im Schultergelenk zu erzielen, während dies bei Verbänden mit herabhängendem Arm wesentlich schwerer, wenn überhaupt möglich, ist.

Neben der Naht bedient sich Herr Prof. Hoeftman bei Kniescheibenbrüchen manchmal eines anderen Verfahrens. Es wird keine Operation vorgenommen, sondern dafür ein besonderer Verband angelegt. Derselbe besteht aus zwei länglichen Streifen aus grobem Heftpflaster, die in der Mitte einen halbkreisförmigen Ausschnitt; in der Grösse der Kniescheibe haben. Rings um diesen Ausschnitt sind Ösen angebracht wie sie an Schnürstiefeln vorhanden sind.

Die Heftpflasterstreifen werden dicht oberhalb und unterhalb der Kniescheibe so angelegt, dass diese in den Ausschnitt hineinpasst, und durch schmälere Heftpflasterstreifen und Umwickeln mit einer Binde in der gewünschten Lage festgehalten. Schliesslich werden die Ösen durch eine Gummischnur zusammengeschnürt, wodurch die Fragmente der Patella aneinander gerückt werden. Verstärkt man die Verschnürung durch einen Bindfaden, kann der Patient sogar herumgehen, da die Fragmente nicht auseinandergesprengt werden können.

Als ferneres Prinzip bei der Behandlung frischer Frakturen der Extremitäten gilt, dass möglichst bald mit Massage und zweckmässigen Bewegungen angefangen wird. Womöglich wird schon am Tage nach der Verletzung mit der Massage angefangen, und mit den Übungen, sobald der Bruch soweit konsolidiert ist, dass bei vorsichtigem Vorgehen ein Wiederbrechen nicht zu befürchten ist. Wie schon $B$ ardenhe uer hervorgehoben hat, ist es kein Zweifel, dass durch ein solches Verfahren die Behandlungsdauer bedeutend verkiirzt und die Resultate besser werden, als wenn die Extremität im festen Verband unbeweglich gelassen wird, bis der Bruch vollständig verknöchert ist.

\section{Uleus cruris.}

Ein Leiden, das bier häutig zur Behandlung kommt, sind die Unterschenkelgeschwüre.

Wenn das Bein geschwollen ist, was meistens der Fall zu sein pflegt, muss der Patient zunächst ein bis zwei Tage zu Bett liegen, bis dio Schwellung zurïckgegangen ist. Dann wird, nachdem das Geschwür selbst mit einem Stück Brandbinde bedeckt worden ist, in üblicher Weise ein Zinkleim verband angelegt. Es ist dabei sorgfältig darauf $z u$ achten, dass keine Ealten sich bilden, weil sonst leicht Druckstellen entstehen. Erst wenn der Verband trocken geworden ist, was einige Stunden dauert, darf der Patient aufstehen.

Die in der Klinik stationär behandelten Patienten müssen ausserdem täglich Übungen im Zander-Institut rornehmen, wobei selbstverständlich solche Apparate ausgeschaltet werden, die eine besondere Bewegung des Fussgelenkes verlangen, oder die einen Druck auf das Ulkus ausüben würden. 
Der Verband wird nach Bedarf durchschnittlich alle 8-14 Tage gewechselt.

Die durch diese Behandlungsmethode gewonnenen Resultate, sind durchaus gut ${ }^{1}$ ).

In solchen Fällen, in welchen neben dem Ulkus noch ein Ekzem besteht, hat sich auch ein Verband mit einer Klebrubinde nach Ferd. von Heuss (Fabrikant 'Teufel) als sebr vorteilhaft erwiesen. Diese Ferbände sind noch leichter anzulegen, als die Zinkleimverbände, und das Geschwür braucht hier nicht bedeckt $z \mathrm{a}$ werden, da die Binde schon für Wundbehandlung präpariert ist.

Diese Verbände können durchschnittlich länger liegen bleiben als die Zinkleimverbände, da sie gegen Feuchtigkeit widerstandsfähiger sind, und daher nur abgewischt $2 u$ werden brauchen, wenn das Sekret an einer Stelle durchkommt.

Es sind hier viele Fälle mit Klebro-

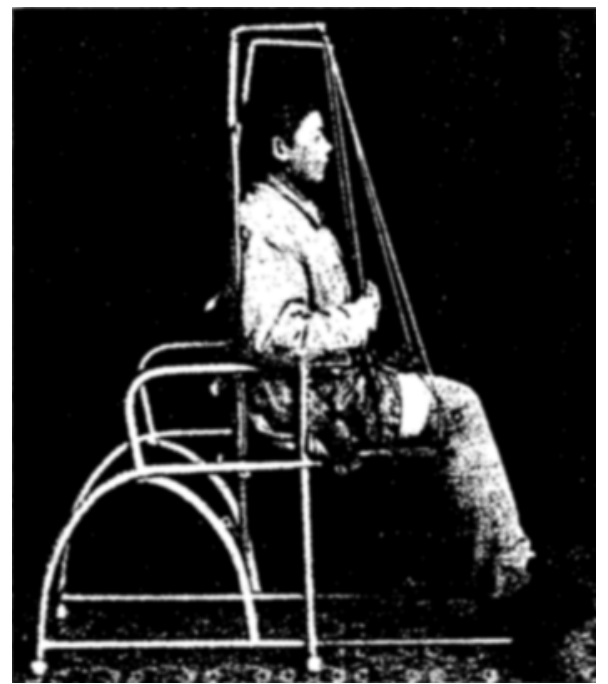

Fig. 55. bindenrerbänden behandelt worden, wobei der Patient während der ganzen Behandlungszeit regelmässig gearbeitet hat und trotzdem eine prompte Heilung erzielt wurde.

\section{Apparat zur Mobilisierung versteifter Hüftgelenke.}

Ebenso wie Herr Prof. Hoeftman früher zwei Apparate zur Beseitigung von Schulterankylosen konstruiert hat ${ }^{2}$, so lat er jetzt auch einen Apparat zur Mobilisierung versteifter Hüftgelenke erfunden ${ }^{3}$ ). Der Apparat (Fig. 55) besteht aus einem Stubl mit rerstellbarer Rückenlehne und einem ebenfalls verstellbaren Fussbrett. Um die Oberschenkel des Patienten werden Gurte angelegt, die nachber mit einer Zugrorrichtung verbunden werden, so dass der Patient selbst mittelst der Hände die Kniee nach aussen oder nach innen zieben kann, während die Füsse feststehen. Hierdurch wirl eine Rotation in den Hüftgelenken bervorgerufen, die bis zu den normalen Grenzen ausgefiihrt werden kann.

Der Apparat hat sich in vielen Fällen als zweckmässig erwiesen.

\section{Röntgenuntersuchungen.}

Vom 1. Januar 1903 bis 1. Januar 1913 sind hier von 6773 Patienten Röntgenanfnahmen gemacht worden.

1) Vergleiche Hoeftman: Beziehungen der Orthopädie zur Arbeitergesetzgebung. Zeitachrift für Orıhopadisehe Chirurgio Band 25.

2) Vergleiche Strube: Bericht über die Hoeftman'sche Klinik.

3) Hoeftman: Verhandl. der Deutschen Gesellschaft für orthopad. Chirugie, III Band. 
Bericht über die Privatklinik des Herro Professor Dr. Hoeftman in Kønigsberg. Pr. 35

Darunter sind selbstverständlich Aufnahmen aller Art vertreten: Nierensteine, Uretheren- und Blasensteine sind häufig nachgewiesen worden. Einmal ist es sogar gelungen, Gallensteine $\mathrm{zu}$ photographieren, was als eine grosse Seltenheit anzusehen ist 1 ).

Ferner wurden die verschiedensten Fremdkörper gefunden und ihre Lage durch stereoskopische Aufnahmen genau festgestellt.

Nicht selten wurden isolierte Frakturen einzelner Hand- und Fusswurzelknochen nachgewiesen, and das Vorkommen derartiger Verletzungen erwies sich als bedeutend häufiger, als man früher ohne Röntgenuntersuchung angenommen hatte.

Nach Luxationen im Schultergelenk zeigte sich sehr oft als Begleitserscheinung eine Verletzung des Tuberculum majus. Aber auch bei Verletzungen der Schulter, die nicht $z \mathfrak{u}$ einer Luxation geführt hatten, wurden sehr oft isolierte Frakturen des Tuberculum majus gefunden. Als typisches Symptom bei derartigen Verletzungen fand sich fast ausnahmslos eine Abmagerung des M. infra- und supraspinatus, sowie eine Behinderung der Auswärtsdrehung des Oberarms. Nieszytka hat in einer Arbeit schon einige Fälle von isolierten Frakturen des Tuberculum majus veröffentlicht ${ }^{2}$ ). Seitdem sind aber noch viele zugekommen. Im ganzen sind in den $10 \mathrm{Jahren} 241 \mathrm{Fer}$ letzungen des Tuberculum majus hier radiographisch festgestellt.

Besonders interessante Aufschlüsse ergab manchmal die Röntgenuntersuchung bei Verletzungen der Halswirbelsäule. Es zeigte sich dann nicht selten, dass eine totale Luxation eines Halswirbels mit starker Verschiebung erfolgen kann, ohne dass Erscheinungen von seiten des Rückenmarks eintreten. Brill $\left.{ }^{3}\right)$ und Malkwitz) baben über derartige, in der Privatklinik des Herrn Prof. Hoeftman beobachtete Fälle berichtet.

Seitdem sind wieder 2 solche Fälle hier beobachtet. Bei dem ersten handelte es sich um einen 56 jährigen Mann, der am 2. August 1912 kopfüber von einem Fuder gefallen war. Nach eigenen Angaben sei er nach dem Fall nicht bewusstlos gewesen, nur habe er nicht atmen können, da sein Kopf ganz nach der linken Brustseite umgebogen gewesen sein soll.

Seine Krau habe dann an dem Kopf gezogen, wonach P. ans dem Munde und aus der Nase geblutet haben soll. Es wurde ihm jedoch dadurch leichter, so dass er nach Hause gehen konnte (1 Kilometer).

Bei der ersten ärztlichen Untersuchung, die schon am Unfalltage stattfand, wurden wohl Hautabschürfungen an der rechten Kopfseite, sowie Schmerzhaftigkeit und Bewegungsbeschränkung der Halswirbelsäule, aber keine Störungen von seiten des Nervensystems gefunden.

Vier Monate später wurde der Verletzte der hiesigen Anstalt überwiesen. Er klagte darüber, dass er bei der Arbeit (ausstrahlende) Schmerzen in der

1) Inaugural-Dissertation von Erno Fett 1906.

2) Inaugura|-Dissertation von Leo Nieszytka. 1906.

3) Inaugural-Dissertation von Friedrich Wilhelm Brill 1911.

4) Inaugaral-Dissertation von Bernhard Malkwitz 1912. (s. dieses Archiv Bd. XI, Heft 4, 1912). 
linken Brustseite und in der linken Hüfte habe; sowie über Unfähigkeit den Kopf richtig zu bewegen.

Die Kopfhaltung des P. war etwas gezwungen, der Kopf schien ein wenig nach vorne verschoben, wodurch die Kontur des Nackens mehr geradlinig verlief als normal; am besten gelang das Beugen nach vorn. Alle anderen Bewegungen waren um etwa die Hälfte beschränkt.

Durch Betasten waren allerdings sichere Veränderungen an der Halswirbelsäule nicht $z u$ finden, im Verein mit der Vorgeschichte liess der Befund jedoch auf eine Wirbelverletzung schliessen, und zwar ergab die Röntgenuntersuchung, dass der Dens Epistrophei abgebrochen und Atlas mitsamt dem Kopf nach vorn luxiert war. Fig. 56.

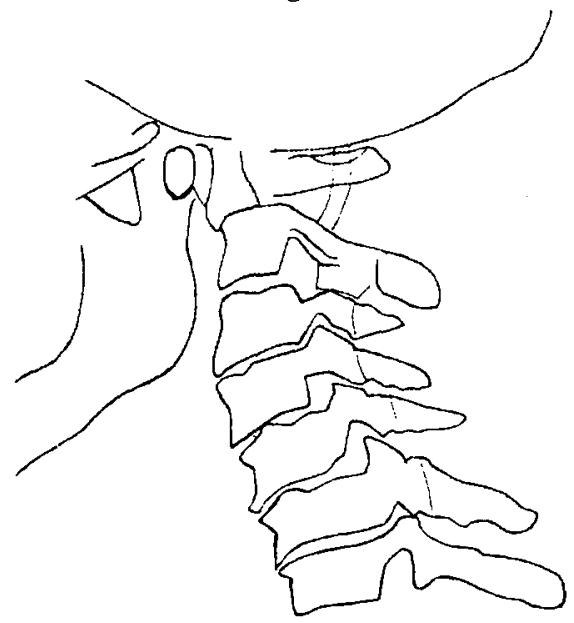

Fig. 56 .

Trotz dieser erheblichen Lageveränderung war das Rückenmark nicht verletat. Es bestanden keine Störungen von seiten der Blase oder des Mastdarmes und keine sonstigen Lähmungen. Las Gefühl war überall erhalten. Vielleicht war die Schmerzempfindung am rechten Arm gesteigert und die mechanische Muskelerregbarkeit hierselbst grösser als links. Die Kniereflexe waren nicht nur nicht gesteigert, sondern sogar sehr schwer auslösbar. Die linke Pupille war ein wenig grösser als die rechte (Sympatikusreiz), also im wesentlichen ein ganz normaler Befund.

Bei dem zweiten Falle bandelte es sich um eine 43 jährige Arbeiterfrau, die am 1. Juli 1912 kopfüber von einem Fuder heruntergefallen war. Sie soll 10 Minuten ohne Besinnung gelegen haben, konnte aber nacbher mit Unterstützung nach Hause gehen (ein Weg von 10 Minuten). Nach 14 Tagen Bettruhe habe sie wieder angefangen, in der Wirtschaft zu arbeiten.

Als sie $1 / 2$ Jahr nach dem Unfall bier aufgenommen wurde, fiel es auf, dass die Kopfhaltung gezwungen war. Der Kopf stand nach vorn verschoben und wurde meistens nach vorne gesenkt gehalten. Frau S. konnte den Kopf nach hinten nur soweit beugen, dass er gerade stand. Das Drehen nach rechts war nur um $10^{\circ}$, nach links um $45^{\circ}$ möglich. 
Bericht über die Privatklinik des Herin Professor Dr. Hoeft ma n in Känigsberg Pr. 37

Beim Betasten des Nackens fühlte man oberhalb des fünften Halswirbels eine Delle, während die Dornfortsätze des sechsten und siebenten Halswirbels deutlich zu fühlen waren.

Der Befund liess auf eine Verletzung im Bereich der Halswirbels:̈ule schliessen, was durch die Röntgenuntersuchung bestätigt wurde. Das Bild (Fig. 5i) zeigt, dass der vierte Halswirbel total nach vorne luxiert und ausser-

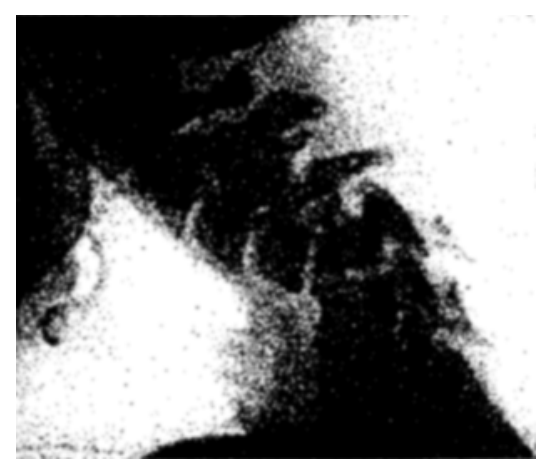

Fig. 57.

dem rechtwinklig ahgeknickt ist, so dass er auf der Vorderseite des fünften Halswirbels liegt.

Wie unter diesen Umständen eine Verletzung des Rückenmarkes hat vermieden werden können, ist schwer zu erklären, ist aber trotzdem Tatsache. Es bestanden keine Lähmungen und das Gefühl war überall erhalten. Nur wäre zu bemerken, dass über dem linken Schulterblatt und über dem linken Pektoralis die Schmerzemptindung als gesteigert angegeben wurde.

Die mechanische Nuskelerregbarkeit war an beiden Armen gleich. Die Bauchdeckenreflexe waren erhalten, die Kniereflexe beiderseits gesteigert ohne Fussklonus.

Wegen der geringen Beschwerden ist auch die Verletzung ron dem zuerst behandelnden Arzte als eine Quetschung aufgefasst worden. Erst die Röntgenuntersuchung zeigte, wie schwer die Veränderungen waren, und die Frau schwebte eigentlich dauernd in Lebensgefahr, bis sie hier eine Kopfstütze bekommen hatte.

Einmal ist sogar eine Halswirbelluxation als Nebenbefund gefunden worden. Es handelte sich damals um eine Verletzung, die nicht im Betriebe passiert und also nicht entschädigungspflichtig war. Der Betreffende erklärte auch, dass er wohl eine gewisse Steitigkeit im Genick hätte, dass sie ihn aber in seiner Arbeit nicht behindere ${ }^{1}$ ). Im ganzen sind 20 Fälle von Halswirbelverrenkungen hier radiographisch nachgewiesen worden.

Auch an anderen Abschnitten der Wirbelsäule zeigte die Röntgenuntersucbung nicht selten Wirbelbrüche, wenn man auch durch die gewöhnlichen

1) Scheutz. Gewöhnung an Cnfallfolgen. Archiv für Orthopadie, Mechanotheropie und Unfallehirurgie Band 12. Helt 1 und 2. 
Untersuchungsmethoden sie nicht nachweisen konnte. - Znweilen wurde bei der Wirbeluntersuchung eine Verknöcherung der Gelenkverbindungen gefunden, und man stand manchmal vor der schwierigen Entscheidung, ob diese Verknöcherung als die Folge eines stattgehabten Traumas anzusehen sei oder

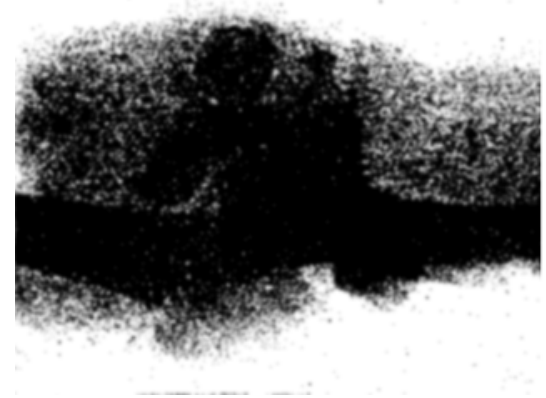

Fig. 58.

Hochgradige Veränderungen im Ellenbogen. gelenk beı Syringomyelie.

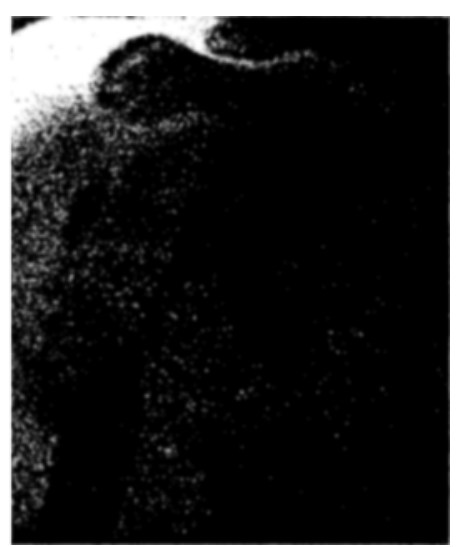

Fig. 59.

Hochgradige Veränderungen im Schultergelenk bei Syringomyelie.

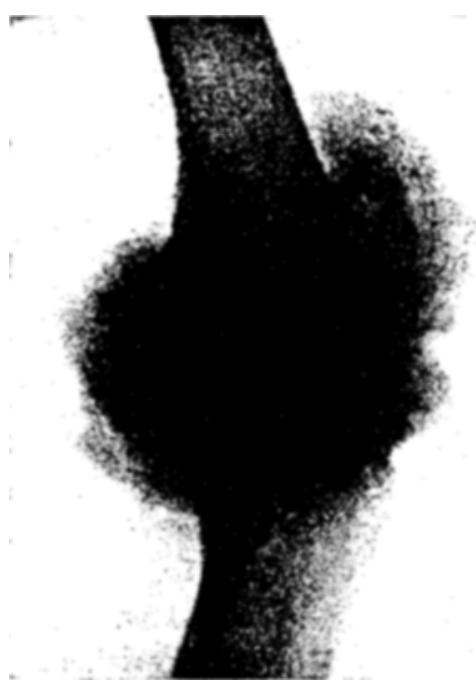

Fig. 60.

Hochgradige Veränderungen im Kniegelenk bei Tabes.

nicht. Auch typische Fälle von Spondylose rhizomélique wurden durch Röntgenphotographie nachgewiesen.

Bei Syringomyelie (Fig. 58 und 59) und Tabes (Fig. 60) zeigten die Röntgenbilder manchmal eine totale Zertrümmerung oder sonstige hochgradige Veränderungen an den Gelenkknochen, ohne dass die Patienten über Schmerzen klagten. 


\section{Muskellähmung ohne Funktionsstörung.}

Yon ungewöhnlichen Fällen, die in hiesiger Anstalt beobachtet wurden, mögen die zwei nachfolgenden noch erwähnt werden. In beiden Fällen handelte es sich um den Ausfall von Muskeln am Schultergürtel, olne dass die Funktion des Armes dadurch gestört war.

Der erste Fall betrifft einen 40jährigen Packer, der am 30. August 1909 rücklings von einem Wagen heruntergefallen war und dabei ausser einer typischen Radiusfraktur eine starke Quetschung der rechten Schulter erlitt, die eine Lähmung des Deltoideus herbeiführte.

Wie das beigefügte Bild (Fig. b1) zeigt, war der rechte Deltamuskel vollstindig atrophisch. Der Armumfang über diesem Muskel gemessen ljetrug

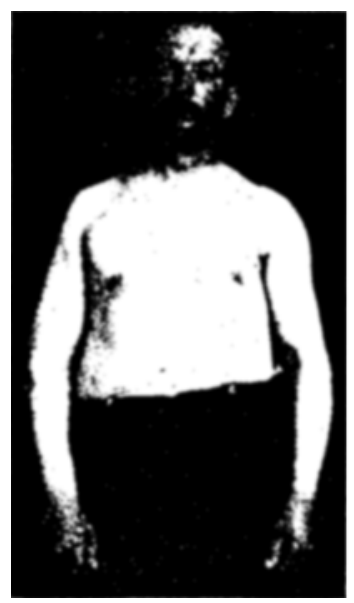

Fig. 61.

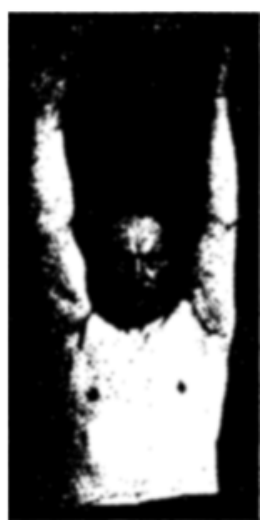

Fig. 62 .

32,5, links 41,j cm. Iagegen war der Ĺmfang über den Bicepls gemessen, beiderseits gleich. Bei der elektrischen Prüfung war an den vorderen und seitlichen Partien des rechten Deltoideus überhaupt keine Reaktion auslösbar. Am hinteren Teil des Muskels war bei sehr starken galvanischen Strömen eine Spur ron Zuckung (Kaz<Anz) nachzuweisen. Es bestanden auch Sensibilitätsstörungen im Ausbreitungsgebiet des $\mathrm{N}$. axillaris.

Es handelte sich also um eine totale Lähmung des Deltamuskels. Trotzdem konnte der Patient, wie Fig. 62 zeigt, den rechten Arm vollkommen normal heben und zwar nach vorne oder nach der Seite in jeder beliebigen Stellung. Er führte das Heben nicht etwa mit einem Schwung aus, sondern er hob den Arm ganz langsam und gleichmässig genan so wie den linken in die Höhe. Es kann dies nur dadurch erklärt werden, dass der Verletzte, der ïberhaupt eine sehr kräftig entwickelte Muskulatur besass, die Hilismnskeln für die Hebung des Armes (supra- und infraspinatus) so geübt hatte, dass sie die Funktion des Deltamuskels übernommen haben.

Der Betreffende arbeitet wie früher als Packer bei einer Speditionsfirma und bekommt denselben Lohn wie andere in seiner Stellung. 
Der zweite Fall betrifft einen 17 jährigen Arbeiter, der in der Kindheit an der linken Brustseite operiert wurde. Über die Anamnese war nichts sicheres zu erfahren, da Patient sich der Vorgänge nur dunkel entsinnnen konnte. Vielleicht hat es sich um ein Empyein gehandelt. Jedenfalls war die Brustmuskulatur an der linken Seite sehr erheblich geschädigt. Vom Pectoralis major war nur die Portio clavicularis und die oberste Ursprungs-

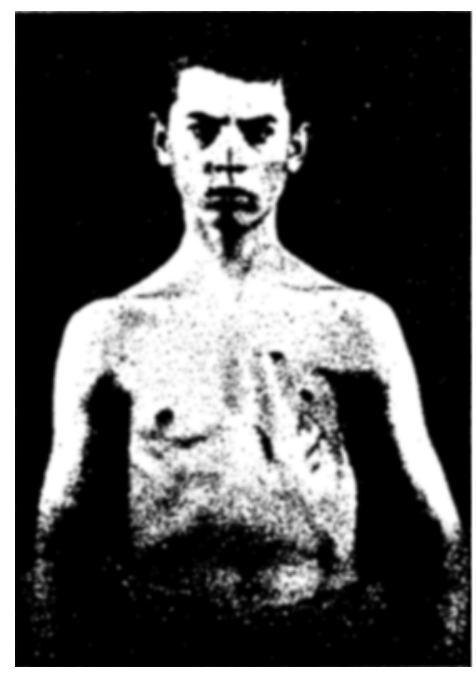

Fig. 63.

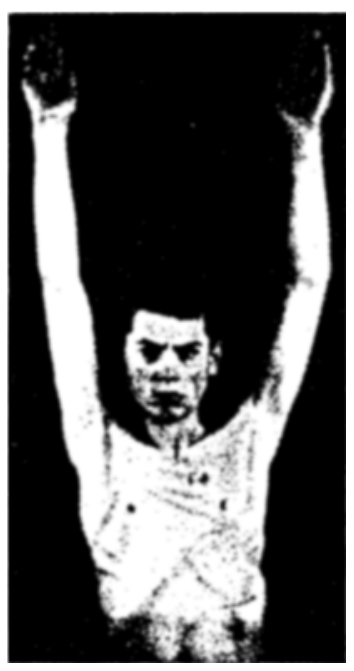

Fig. 64 .

zacke von Stermum, von Pectoralis minor nur die Crsprangszacke von der dritten Rippe erhalten (Fig. 63). Auch von Serratus anticus major fehlten einige von den oberen Ursprungszacken. Trotzdem konnte der linke Arm vollkommen frei bewegt werden (Fig. 64) und die Kraft desselben war nicht herabgesetzt. M. führte an den Zander-Apparaten sämtliche Übungen mit den schwersten Widerständen aus.

\section{Simulation von Unfallfolgen.}

Jeder, der mit Unfallverletzten zu tun hat, muss sich auch notgedrungenermassen mit der Simulation von Unfallfolgen beschäftigen. Wenn es sich um einen entschädigungspflichtigen Unfall handelt, wird man äusserst selten erleben, dass die Patienten erklären, dass sie keine Beschwerden mehr haben. Wenn aber auch die Übertreibung auf der Tagesordnung steht, so ist die reine Simulation doch verhältnismässig sebr selten. Noch viel schwieriger ist es in den meisten Fällen, die Simulation einwandfrei nachzuweisen. Es genügt nicht nur, dass man selbst die moraliche Überzeugung hat, dass der Patient simuliert, man muss auch Gründe anfübren können, aus denen auch der Laie ersehen kann, dass Simulation vorliegt. Und dies ist manchmal nicht möglich. Es gebört hierzu ausser genügender Erfahrung ein sehr genaues Aktenstudium und eine eingehende Untersuchung und Beobachtung des Patienten. 
Bericht über die Privatklinik des Herrn Professor Dr. Ho eftman in Königsberg Pr. 41

Im folgenden sollen einige Fälle von Simulation angeführt werden, die hier beobachtet und nachgewiesen sind.

Der erste Fall betrifft einen 33jährigen Mann, der im August 1905 im Bergwerk von einem Kohlenwagen von hinten angefahren wurde, so dass er vornüber hinfiel und eine Quetschung am linken Fuss und am Kreuz erlitt. Nach eigenen Angaben des Verletzten soll er 8 Tage nach dem Unfall, als er im Krankenhause Bergmannsheil behandelt wurde, Blat gehustet haben. Hiervon ist aber in den diesbezüglichen Gutachten nichts enthalten. Wohl aber geht aus denselhen hervor, dass der Betreffende schon vor dem Unfall Neurastheniker war.

Bei seiner Entlassung aus dem Krankenhause Bergmannsheil waren objektive Unfallfolgen nicht nachzuweisen, es wurde aber eine Gewöhnungsrente von $33 \frac{1}{3} \%$ vorgeschlagen, weil es angebracht erschien, duss W. sich eine Zeitlang schonte.

Als W. ein Jahr später der hiesigen Anstalt zur Untersuchung überwiesen wurde, klagte er über eine Reihe Beschwerden, vor allem aber über Luftmangel und dauernde Schmerzen in der Brust. Die sorgfältige, anch von spezialistischer Seite wiederholt vorgenommene Lungenuntersuchung liess jedoch keinen Anhalt für seine Klatgen finden. In dem sehr spärlichen Auswurf waren auch nach Verabreichung ron Jodkali keine Tuberkelbazillen nachzuweisen. Trotzdem wurde W. angeblich ron Tag zu Tag schwächer, bis er schliesslich erklärte, so elend zu sein, dass er zu Bett liegen müsse. Die an diesem Tage in der Achselhöhle gemessene Körpertemperatur ergab $36^{\circ} \mathrm{C}$.

In den folgenden Tagen zeigte das Thermometer bei jeder Messung eine Steigerung der Temperatur, bis es am Abend des vierten Tages anf $37,9^{\circ}$ lram. Eine unmittelbare Nachmessung ergab 37,7 ${ }^{\circ}$. Da dies bei dem sonst völlig negativen Befunde eigentümlich erschien, wurde sofort zum dritten Male eine Kontrollmessung vorgenommen und $W$. während derselben genan beobachtet, was bei den rorhergehenden nicht der Fall war. Das Thermometer zeigte jetzt nur $36,8^{\circ}$. Auch in den nächsten Tagen war die Temperatur vollkoinmen normal.

Es lässt dies nur den Schluss zu, dass W. durch irgendwelche Manipulationen (z. B. Reiben des Thermometers) das Quecksilber zum Steigen gebracht hatte, um Fieber rorzutäuschen.

Als dies dem W. gesagt wurde, verlangte er seine Entlassung, die dann auch unmittelbar erfolgte. Fr konnte dann auch mit einem Male ganz anstandslos aufstebion und sich frei bewegen, obwohl er kurz vorher erklärte, dass er so elend soi, dass er sich im Bett kaam rühren könne.

Ein ähnlicher Fall wurde im Mai 1909 beobachtet. Es handelte sich damals um eine Frau, die wegen einer Armverletzung in die Klinik kam. Nachdem sie schon einige Tage im Zander-Institut Übungen vorgenommen, die Bewegungen aber sehr mangelhaft und nur mit ganz kleinen Widerständen ausgeführt, sollte versucht werden, ob sie nicht imstande wäre, die Bewe- 
gungen besser auszuführen. Gleich am ersten Apparat fing sie aber derartig zu jammern an, dass ron einer Prüfung überhaupt Abstand genommen wurde und die Frau auf ihr Zimmer geschickt werden musste. Hier setzte sie ihre Lamentationen fort und warf sich schliesslich auf die Erde, worauf sie zu Bett gebracht wurde.

Am nächsten Tage war sie angeblich unfähig aufzustehen. Bei der Messung der Körpertemperatur zeigte das Thermometer zunächst $40^{\circ} \mathrm{C}$, während die gleich darauf - allerdings unter dauernder Aufsicht - vorgenommene Kontrollmessung eine normale Temperatur von $37^{\circ}$ ergab.

Ein Oberinspektor fiel im Dezember 1905 eine Treppe herunter und verletzte dabei das linke Knie. Uber den Befund unmittelbar nach dem Lnfall liegen keine sicheren Angaben vor. Der erste ärztliche Befund datiert sich 3 Wochen nach der Verletzung und zwar wurde danach angenommen, dass es sich ausser einer starken Quetschung auch noch um einen schlecht verheilten Bruch der Kiniescheibe handelte.

Der Betreffende war in einer Privatversicherungsgesellschaft versichert und bekam von derselben, da eine Versteifung des Kniegelenkes angeblich nach dem Unfall eingetreten war, eine Abfindungssumme ron 6000 Mark. Da aber der Unfall im landwirtschaftlichen Betriebe passiert war, wurde er ausserdem noch lange Zeit ron der Berufsgenossenschaft durch eine Rente entschädigt, die zuletat auf $40 \%$ festgesetzt war.

Im Oktober 1908 ging nun bei der Berufsgenossenschaft ein Schreiben des Inbaits ein, dass der Betreffende gar kein steifes Bein habe. Vielmehr sei bei einer etwas ausgiebigen Feier beobachtet worden, wie der Betreffende zu gleichen Füssen von der Erde auf einen Tisch gesprungen wäre.

Auf Grund dieses Schreibens ordnete die Berufsgenossenschaft eine Untersuchung in hiesiger Anstalt an. Es wurde dabei wahrgenommen, dass der Verletzte sein linkes Knie steif hielt unter Angabe, dass er das Kniegelenk so gut wie gar nicht beugen könne. Versuchte man dies passiv zu tun, spannte er sofort seine Beinmuskulatur so an, dass ein Beugen unmöglich war. Er gab auch an, dass das Knie äusserst empfindlich wäre.

Die Röntgenuntersuchung ergab einen absolut normalen Befund. Es war aus dem Bilde mit völliger Sicherheit auszuschliessen, dass ein Bruch der Kniescheibe oder überhaupt eine Knochenverletzung vorgelegen hatte. Auch der sonstige Befund war negativ. Die Konturen des linken Knies waren denen des rechten rollkommen gleich. Es bestand kein Erguss und anch keine erböhte Temperatur am verletzten Knie. Vor allem aber bestand keine Abmagerung des linken Beines. Die Cmfangsmasse der Oberschenkel waren beiderseits gleich. Die linke Wade sogar $1 \mathrm{~cm}$ stärker als die rechte.

Nach dem ganzen Befunde konnte nur angenommen werden, dass der Verletzte willkürlich sein Bein steif hielt. Er wurde daher auf einen so hohen Sitz gesetzt, dass er mit den Füssen den Fussboden nicht erreichen konnte. Dabei beugte sich allmählich das linke Knie bis zum rechten Winkel. Es wurde nun noch ein Gummizug angelegt, wodurch das Bein bis zum Ex- 
tremen sich beugen liess. Der Betreffende war allerdings über diese schnelle „Heilung“ wenig erfreut.

Der Arbeiter P. aus J. kam im Jahre 1911 wegen einer rechtsseitigen Hüftgelenkserkrankung zur Lntersuchung in die hiesige Anstalt. Er gab an, dass der Zustand seines rechten Beines durch einen Unfall im Oktober 1903 hervorgerufen wäre. Er will damals beim Aufrichten von einem Kartoffelsack einen plötzlichen Schmerz in der rechten Hüfte verspürt haben. Er beziehe auch hierfür eine Lnfallrente.

Die Untersuchang ergab, dass das rechte Bein um ca $4 \mathrm{~cm}$ verkürat und der rechte Oberschenkel um $7 \mathrm{~cm}$ abgemagert war. Das Bengen im rechten Hültgelenk gelang nur bis zum rechten Winkel, die Rotation war so gut wie aufgehoben.

Die Röntogenuntersuchung zeigte, dass es sich um eine deformierende Erkrankung des rechten Hüftgelenkes handelte. Ausserdem aber waren am Schenkelhals Veränderungen rorhanden, die den Eindruck eines verheilten Schenkelhalsbruches machten. Somit schien zunächst der Zusammenhang mit dem Unfall einwandsfrei. Beim Durchlesen der Akten fand sich aber eine kurze Notiz, dass P. im Jahre 1901, also zwei Jahre vor dem Unfall, wegen einer rechtsseitigen Hüftgelenkserkrankung einen Antrag auf Invalidenrente gestellt hatte. Hierüber hefragt, leugnete P., dass sein rechtes Bein vor dem Unfall erkrankt gewesen wäre und blieb hartnäckig bei dieser Angabe, obwohl er wiederholt auf die Unrichtigkeit seiner Angaben aufmerksam gemacht wurde.

Aus den Akten der Landesversicherungsanstalt aus dem Jahre 1901 ging nämlich einwandsfrei hervor, dass der damalige Befund genau derselbe war, wie bei der diesseitigen Untersuchung im Jahre 1911. Es bestand schon damals dieselbe Verkürzung, Bewegungsbeschränkung und Abmagerung des rechten Beines wie jetzt.

Es ist also klar, dass P. gegen besseres Wissen den Zustand des rechten Beines auf den Unfall zurückzuführen versuchte, während in der Tat das Trauma, wenn überhaupt von einem solchen gesprochen werden kann, jedenfalls keine Folgen hinterlassen hatte:

Es seien hier ferner zwei Fälle erwähnt, die eine grosse $̈$ hnlichkeit mit einander aufweisen. Sie waren beide im November 1908 rom Schiedsgericht zur Beobachtung hierher geschickt, und in beiden Fällen hatte es sich um eine Schulterverletzung gehandelt, die angeblich eine erhebliche Bewegungsbeschränkung des Armes zurückgelassen hatte. Wie gross dieselbe angeblich war, geht aus den Figuren 65 und 66 am besten herror.

In beiden Fällen konnte aber einwandsfrei nachgewiesen werden, dass die Betreffenden die Arme wesentlich besser heben und gebrauchen konnten, als sie angaben. Es gelang nämlich, die Patienten so zu photographieren, wie die Figuren 67 und 68 zeigen, wobei za bemerken ist, dass die Betreffenden den verletzten Arm ohne jegliche Hilfe oder Unterstuitzung selbsttätig so hoch gehoben, wie die Bilder zeigen. 
Nan muss nur bei einem solchen Versuch den Mann den Zweck nicht merken lassen und vor allem das Wort "heben" sorgfältig vermeiden.

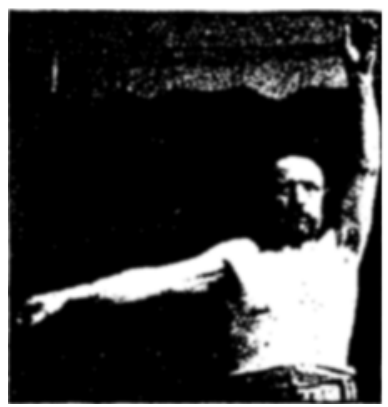

Fig. 65.

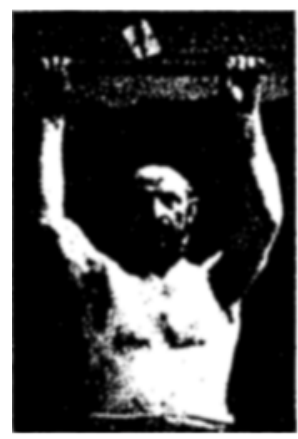

Fig. 67.

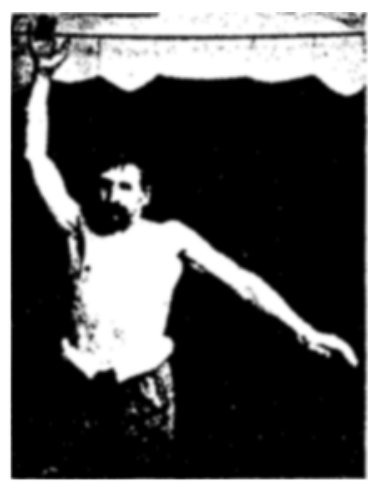

Fig. 66 .

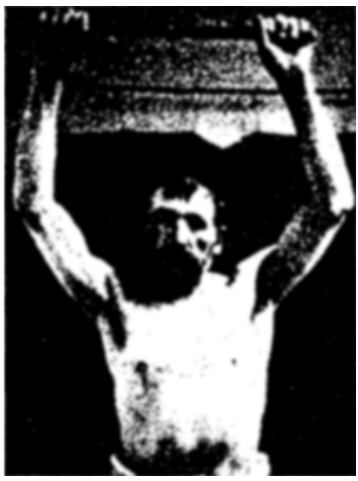

Fig. 68.

Interessant ist der Umstand, dass in beiden Fällen die Bilder 67 und 68 zuerst gemacht wurden. Erst nachher wurden die Photographien 65 und 66 aufgenommen, nachdem dem Patienten gesagt war, dass festgestellt werden sollte, wie hoch er den Arm heben könne.

Zum Seliluss sei noch ein besonders krasser Fall von Simulation erwähnt. Es handelte sich um einen Zimmermann, der nach der Unfallanzeige 1896 eine Quetschung der Brust erlitten hatte. Später findet sich in den Akten die Angabe, dass W. bei dem Unfall den gebeugten rechten Arm gegen die Brust gehalten, und also auch eine Quetschung des rechten Unterarmes erlitten haben soll.

Während nun in dem Gutachten zunächst nur ron der Brustquetschung gesprochen wird, die jedoch objektiv nachweisbare Folgen nicht hinterlassen hatte, wurde nach einiger Zeit ein Zittern des rechten Armes gefunden, das als Unfallfolge durch eine Rente von $75 \%$ entschädigt wurde. Diese Rente hat W. dann jahrelang bezogen. Zwar war im Laufe der Zeit der Verdacht 
entstanden, dass W. simulierte, und die Berufsgenossenschaft hatte eine Beobachtung durch den Ortsgendarmen angeordnet, aber ohne ein positives Resultat.

Als W. dann 1903 in die biesige Anstalt zur Untersuchung kam, fiel es auf, dass er meistens die rechte Hand in der Tasche hielt, angeblich weil er sich genierte, seine zittrige Hand zu zeigen.

Da es sich nun darum bandelte, festzustellen, ob es sich um Paralysis agitans oder um Simulation handelte, wurde W. am nächsten Morgen in ein Zimmer untergebracht, wo er dauernd unter Aufsicht blieb. Er durfte auch die rechte $H_{a}$ and nicht in der Tasche halten. Es fiel dabei auf, dass er mit der Hand zwar zitterte, aber in verschiedener Weise, indem er bald Pround Supinationsbewegungen ausführte, bald aber mit Beuge- und Streckbewegungen im Handgelenk abwechselte. Manchmal schien er zu ermüden und machte dann mit dem ganzen Arm Streck- und Beugebewegungen nach oben und unten.

Als er in dieser Weise 8 Stunden gesessen hatte, war er anscheinend zu Ende mit seiner Kraft und verlangte nun seine Entlassung, die dann auch erfolgte. Vorher musste er aber ein Protokoll unterschreiben, dass er auf eigenen Wunsch die Klinik verliesse. Er brauchte zu dieser Unterschrift eine sehr erhebliche Zeit. Der Name war trotzdem fast unleserlich, während ein in den Akten befindliches, kurze Zeit vorher von W. an die Berufsgenossenschaft gerichtetes Schreiben eine gate leserliche Schrift zeigte.

Als W. nachher die Klinik verliess, wurde eine ibm unbekannte Person nachgeschickt, die auf dem Bahnhof beobachtete, wie W. mit der rechten Hand ein Glas Bier an den Mund führen konnte, ohne etwas zu vergiessen.

In dem darauf abgegebenen Gutachten wurde dann ausgeführt, dass eine Erwerbsbeschränkung infolge des Unfalls nicht anzunehmen wäre.

Nachdem später der Amtsrorsteher in der Heimat des W. bekundete, dass dieser als Tischler arbeitete, ohne dass eine Erwerbsbeschränkang zu bemerken wäre, hob das Schiedsgericht die Rente von $75 \%$ auf.

Etwa ein halbes Jahr später kam dann ein Gutsbesitzer aus der Gegend des W. als Patient in die Kilinik und erzähite zufällig eines Tages, dass W. zu ihm gekommen wäre und sich angeboten hätte, als Tischler gegen einen Tagelohn von einer Mark zu arbeiten, während er früher 4 Mark verdient bätte. Er hatte sich auch hierüber eine Bescheinigung geben lassen.

Auf diese Weise hatte W. sich von den Gutsbesitzern in der ganzen Gegend bescheinigen lassen, dass er wegen Zittern in der rechten Hand nur ein Viertel gegen früher verdiene und wollte diese Bescheinigungen für einen Rekurs beim Reichsversicherungsamt benutzen.

Er wurde aber nun von einer Person in seiner Heimat denunziert und zwar schrieb die betreffende, dass W., der angeblich inmer mit der rechten Hand zittern müsse, ein viel beschäftigter Schreiber wäre.

W. wurde über diese Anzeige so erbost, dass er dem Denunzianten eine Scheune in Brand steckte, worauf er wegen Brandstiftung ins Gefängnis kam. Bevor seine Verurteilung erfolgte, erhängte er sich. 


\section{Bemerkungen über einige Arzneinittel.}

Zuletzt möchte ich über einige Arzneimittel berichten, die in der Privatklinik des Herrn Prof. Hoeftman öfters und mit gutem Resultat zur Verwendung kommen.

Um dann mit dem Radium zu beginnen, ist seit 1909 ein Trinkapparat hier aufgestellt. Es sind aus demselben über 7000 Portionen abgegeben, die sich auf 155 Patienten verteilen. Der Gesamteindruck ist der, dass das Mittel bei Gicht and rheumatischen Erkrankungen günstig wirkt. Sogar in schweren Fällen, die jahrelang mit anderen Mitteln ohne Erfolg belandelt worden sind, ist Besserung resp. Heilung erzielt worden.

Anfänglich warden auch Radiambäder verordnet; jetzt. kommen keine solche mehr bier zur Verwendung; da die Resultate weder den Kosten, noch den Erwartungen entsprachen.

Bei Gelenkversteifungen oder Narbenkontrakturen nach Phlegmonen werden gleichzeitig mit der mediko-mechanischen Behandlung Einspritzungen von Fibrolysin vorgenommen. Nach den hier gemachten Erfahrungen wirkt das Mittel günstig. Direkt erwiesen ist dies z. B. in einem Fall von hochgradiger Fingerversteifung. Es handelte sich um einen Nann, der schon monatelang mediko-mechanisch behandelt war, ohne dass ein Erfolg erzielt wurde. Als er aber dann Fibrolysin bekam, trat in kaum drei Wochen eine solche Besserung ein, dass Patient die Hand vollständig zur Faust schliessen konnte.

Bevor das Fibrolysin zu haben war, wurde hier mehrfach das Thiosinamin verwandt. Es wurde aber davon Abstand genommen, da das Mittel manchmal unangenehme Begleiterscheinungen zeigte. So bekam z. B. ein Patient, der früher ein Erysipel gehabt batte, nach dem Thiosinamin wieder ein solches. Ein anderer bekam Bluthusten und ein dritter zeigte delirienartige psychische Störungen. Allerdings gingen die Erscheinungen nach Aussetzen der Einspritzungen zurück. Obwohl das Fibrolysin nur ein Derivat von dem Thiosinamin ist, sind nach seinem Gebrauch störende Begleiterscheinungen hier nie beobachtet worden.

Ein Mittel, das sich hier bewährt hat, ist das Aristochin. Es muss allerdings zugegeben werden, dass bei Erkältungskrankheiten das Antifebrin, in einer Gabe von $0,25 \mathrm{~g} 2-3 \mathrm{mal}$ täglich gegeben, wirksamer ist. Immerhin ist auch hier das Aristochin von grossem Vorteil, wenn es möglichst gleich im Anfang gegeben wird, da dadurch häufig die ganze Krankheit kupiert wird. Ausserdem ist es als ein Chininderivat weniger schädlich für das Herz.

Vor allem aber hat sich dieses Mittel als ein Spezifikum bei Zahnschmerzen erwiesen, sowohl bei einfachen neuralgischen Erkrankungen, als auch bei durch Karies hervorgerufenen Schmerzen. In diesem Falle genügt fast immer eine Tablette von $0,25 \mathrm{~g}$ zu zerkauen und den dadurch entstandenen Brei in die Gegend der kranken, schmerzbaften Stelle mit der Zunge einzureiben. Ebenso wirksam ist dies bei Wurzelaffektionen, die etwa nach Plombierungen Schmerzen hervorrufen. 
Von äusseren Mitteln sei die Lenizetsalbe erwähnt. Bei Pusteln, beginnenden Farunkeln an behaarten Stellen (Achselhöhle), sowie bei Röntgenverbrennungen sind gute Resultate hiermit erzielt worden. Ferner ist es bei "Wolf" oder bei durch Schweiss bedingten Ulzerationen zwischen den Zehen, oder bei Fettleibigen in der Schenkelbeuge oder Ellenbogenbenge, zu empfehlen. Gute Dienste leistet das Mittel auch bei Ekzem an resp. in der Nase. Es gelingt manchmal rot entzündliche Stellen auf der äusseren Nasenhant dadurch zu beseitigen, dass die Lenizetsalbe in die Nase eingestrichen wird, und dort bestehende Reizzustände beseitigt.

Schliesslich sei auf das alte Mittel, das Thymol, hingewiesen. Namentlich ist es bei Quetschwunden zu empfehlen. Auch wenn nach Quetschungen infektiöse Zustände eintreten, lässt sich manchmal durch Anwendung von Thymol-Umscblägen $(1: 1000)$, die allerdings mindestens täglich erneuert werden müssen, sehr gute Resultate erzielen.

Ausserdem wirkt das Mittel bei Geschwären sehr schnell und gut. Nur hat es einen Nachteil, nämlich dass die Wunden leicht schmerzhaft werden.

\section{Schlusswort.}

Aus dem vorstehenden Bericht dürfte wohl hervorgehen, dass neben der Behandlung von Unfallverletzten das charakteristische für die Privatklinik die Anfertigung von Apparaten, besonders Prothesen, ist. In bezug auf die letzteren dürfte das Jahr 1913 vielleicht einen dbschnitt bedeuten, da im Januar dieses Jahres hier in Königsberg eine Krüppel-Heil- und Lehranstalt eingerichtet wurde, die ebenfalls unter der Leitung des Herrn Prof. Hoeftman steht. Voraussichtlich werden nun viele Fälle, die bis dahin in seiner Privatklinik behandelt wurden, von jetzt $a b$ in der neuen Anstalt untergebracht werden. Für den Gesamtbetrieb in der hiesigen Klinik wird dies jedoch nicht von einschneidender Bedeutung sein.

Zuletzt möchte ich nicht verfehlen, meinem verehrten Chef, Herrn Prof. Hoeftman, für die Überlassung des Materials meinen Dank auszusprechen. Auch Herrn Dr. Matthias, der mir die Röntgenphotographien zur Verfügung gestellt hat, sei an dieser Stelle gedankt. 